TRANSACTIONS OF THE

AMERICAN MATHEMATICAL SOCIETY

Volume 363, Number 3, March 2011, Pages 1419-1443

S 0002-9947(2010)04784-1

Article electronically published on October 20, 2010

\title{
BLOW-UP RATE OF TYPE II AND THE BRAID GROUP THEORY
}

\author{
NORIKO MIZOGUCHI
}

\begin{abstract}
A solution $u$ of a Cauchy problem or a Cauchy-Dirichlet problem for a semilinear heat equation$$
u_{t}=\Delta u+u^{p}
$$

with nonnegative initial data $u_{0}$ is said to undergo type II blow-up at $t=T$ if

$$
\limsup _{t \nearrow T}(T-t)^{1 /(p-1)}|u(t)|_{\infty}=\infty
$$

Let $\varphi_{\infty}$ be the radially symmetric singular steady state of the Cauchy problem. Suppose that $u_{0} \in L^{\infty}$ is a radially symmetric function such that $u_{0}-\varphi_{\infty}$ and $\left(u_{0}\right)_{t}$ change sign at most finitely many times. By application of the braid group theory, we determine the exact blow-up rate of solution with initial data $u_{0}$ which undergoes type II blow-up in the case of $p>p_{J L}$, where $p_{J L}$ is the exponent of Joseph and Lundgren.
\end{abstract}

\section{INTRODUCTION}

This paper is concerned with the blow-up rate of solutions to a Cauchy problem for a semilinear heat equation

$$
\begin{cases}u_{t}=\Delta u+u^{p} & \text { in } \mathbf{R}^{N} \times(0, T) \\ u(x, 0)=u_{0}(x) \geq 0 & \text { in } \mathbf{R}^{N}\end{cases}
$$

and a Cauchy-Dirichlet problem

$$
\begin{cases}u_{t}=\Delta u+u^{p} & \text { in } \Omega \times(0, T), \\ u(x, 0)=0 & \text { on } \partial \Omega \times(0, T), \\ u(x, 0)=u_{0}(x) \geq 0 & \text { in } \Omega\end{cases}
$$

in a domain $\Omega \subset \mathbf{R}^{N}$, where $p>1, T>0$ and $u_{0} \in L^{\infty}$. Here a solution $u$ of (1.1) or (1.2) is said to blow up at $t=T<\infty$ if $\limsup _{t \nearrow T}|u(t)|_{\infty}=\infty$ with the supremum norm $|\cdot|_{\infty}$. It is well known by 4 that if $p$ is subcritical in the Sobolev sense, then any solution of (1.1) or (1.2) in a convex domain blowing up at $t=T$ fulfills

$$
|u(t)|_{\infty} \leq C(T-t)^{-\frac{1}{p-1}} \quad \text { for } t \in[0, T)
$$

with some constant $C>0$. The blow-up satisfying (1.3) is said to be of type I and of type II otherwise.

Received by the editors July 2, 2007 and, in revised form, May 15, 2009.

2000 Mathematics Subject Classification. Primary 35K20, 35K55.

(C)2010 American Mathematical Society 
In the supercritical case, i.e., $p>p_{S}$ with the Sobolev critical exponent $p_{S}$, there seem to be known results only for radially symmetric solutions. If $u$ is radially symmetric, then (1.1) and (1.2) are written as

$$
\begin{cases}u_{t}=u_{\xi \xi}+\frac{N-1}{\xi} u_{\xi}+u^{p} & \text { in }(0, \infty) \times(0, T), \\ u(\xi, 0)=u_{0}(\xi) \geq 0 & \text { in }[0, \infty)\end{cases}
$$

and

$$
\begin{cases}u_{t}=u_{\xi \xi}+\frac{N-1}{\xi} u_{\xi}+u^{p} & \text { in }(0, c) \times(0, T), \\ u(c, 0)=0 & \text { in }(0, T), \\ u(\xi, 0)=u_{0}(\xi) \geq 0 & \text { in }[0, c]\end{cases}
$$

with $c>0$, respectively, where $\xi=|x|$. Let $\varphi_{\infty}$ be the singular steady state of (1.4) defined by

$$
\varphi_{\infty}(\xi)=c_{\infty} \xi^{-\frac{2}{p-1}} \quad \text { for } \xi>0
$$

with

$$
c_{\infty}=\left\{\frac{2}{p-1}\left(N-2-\frac{2}{p-1}\right)\right\}^{\frac{1}{p-1}} .
$$

Let $p_{J L}$ be the exponent of Joseph and Lundgren, i.e.,

$$
p_{J L}= \begin{cases}\infty & \text { if } N \leq 10, \\ 1+\frac{4}{N-4-2 \sqrt{N-1}} & \text { if } N \geq 11 .\end{cases}
$$

For a function $f$ on $[0, \infty)$ with $f \neq \equiv 0$, let $z(f)$ be the supremum over all $k$ such that there exist $0 \leq r_{1}<r_{2}<\cdots<r_{k+1}<\infty$ with $f\left(r_{i}\right) \cdot f\left(r_{i+1}\right)<0$ for $i=1,2, \ldots, k$. It was shown in [11] that type II blow-up does not occur for any solution of (1.4) with $z\left(u_{0}-\varphi_{\infty}\right), z\left(\left(u_{0}\right)_{t}\right)<\infty$ or of (1.5) in the case of $p_{S}<p<p_{J L}$. On the other hand, when $p>p_{J L}$, there exists a type II blow-up solution of (1.4) by [6], 7]. The blow-up rate of their solution is some negative power of $T-t$ with blow-up time $T$. The author obtained type II blow-up solutions to (1.5) by a different method for $p>1+7 /(N-11)$ and $N \geq 12$ in [16]. The method can be easily modified to the Cauchy problem (1.4). There were no results on detailed blow-up rate of type II blow-up solutions except [6], 7] before [17.

Putting

$$
w(r, s)=(T-t)^{\frac{1}{p-1}} u(\xi, t)
$$

with $r=(T-t)^{-1 / 2} \xi$ and $s=-\log (T-t)$ for a solution $u$ of (1.4), $w$ satisfies

$$
\begin{cases}w_{s}=w_{r r}+\frac{N-1}{r} w_{r}-\frac{r}{2} w_{r}-\frac{1}{p-1} w+w^{p} & \text { in }(0, \infty) \times\left(s^{T}, \infty\right), \\ w\left(r, s^{T}\right)=T^{1 /(p-1)} u_{0}\left(T^{1 / 2} r\right) & \text { in }[0, \infty),\end{cases}
$$


where $s^{T}=-\log T$. When $u$ is a solution of (1.5), $w$ defined in (1.7) fulfills

$$
\begin{cases}w_{s}=w_{r r}+\frac{N-1}{r} w_{r}-\frac{r}{2} w_{r}-\frac{1}{p-1} w+w^{p} & \text { in } \underset{s^{T}<s<\infty}{\bigcup}\left(\Omega_{s} \times\{s\}\right), \\ w(r, s)=0 & \text { on } \bigcup_{s^{T}<s<\infty}\left(\partial \Omega_{s} \times\{s\}\right), \\ w\left(r, s^{T}\right)=T^{1 /(p-1)} u_{0}\left(T^{1 / 2} r\right) & \text { in } \Omega_{s^{T},}\end{cases}
$$

where $\Omega_{s}=e^{s / 2}[0, c)$.

We note that $\varphi_{\infty}$ is also a singular steady state of (1.8). Let $L_{\rho}^{q}$ be the class of radially symmetric functions $f$ on $\mathbf{R}^{N}$ with $\int_{0}^{\infty}|f(r)|{ }^{q} r^{N-1} \rho(r) d r<+\infty$ for $q \geq 1$, and let $H_{\rho}^{1}=\left\{f \in L_{\rho}^{2}: f^{\prime} \in L_{\rho}^{2}\right\}$, where $\rho(r)=\exp \left(-r^{2} / 4\right)$ for $r \geq 0$. We consider an eigenvalue problem

$$
\phi^{\prime \prime}+\frac{N-1}{r} \phi^{\prime}-\frac{r}{2} \phi^{\prime}-\frac{1}{p-1} \phi+\frac{p c_{\infty}^{p-1}}{r^{2}} \phi=-\lambda \phi \quad \text { in } H_{\rho}^{1} .
$$

The left-hand side is the linearized operator of (1.8) at $\varphi_{\infty}$. The following was shown in [6, 7] (also see 14]): If $p>p_{J L}$, then the spectrum of (1.10) consists of countable eigenvalues and the $j$ th eigenvalue $\lambda_{j}$ is given by

$$
\lambda_{j}=\frac{\alpha}{2}+\frac{1}{p-1}+j \quad \text { for } j=0,1,2, \ldots,
$$

where

$$
\alpha=\frac{-(N-2)+\sqrt{\beta^{2}-4(p-1) c_{\infty}^{p-1}}}{2}
$$

with $\beta=N-2-4 /(p-1)$. The $j$ th eigenfunction $\phi_{j}$ normalized in $H_{\rho}^{1}$ with $\phi_{j}(r)>0$ near $r=0$ behaves as

$$
\phi_{j}(r)=c_{j} r^{-|\alpha|}+o\left(r^{-|\alpha|}\right) \quad \text { as } r \rightarrow 0
$$

and

$$
\phi_{j}(r)=\widetilde{c}_{j} r^{2 \lambda_{j}-\frac{2}{p-1}}+o\left(r^{2 \lambda_{j}-\frac{2}{p-1}}\right) \quad \text { as } r \rightarrow \infty
$$

with some constants $c_{j}>0$ and $\widetilde{c}_{j}$. We note that $\lambda_{0}<\lambda_{1}<0$ for $p>p_{J L}$ and that $\lambda_{2}>0$ for $p>p_{L}$, where $p_{L}$ is the Lepin exponent, i.e.,

$$
p_{L}= \begin{cases}\infty & \text { if } N \leq 10, \\ 1+\frac{6}{N-10}\left(>p_{J L}\right) & \text { if } N \geq 11 .\end{cases}
$$

According to 12, if $w$ is a global solution of (1.8) or (1.9) corresponding to a type II blow-up solution, then $w(s) \rightarrow \varphi_{\infty}$ in $H_{\rho}^{1}$ and in $C_{l o c}^{1}(0, \infty)$ as $s \rightarrow \infty$. The readers can find its simple proof in [17, which works under the hypotheses of our theorems below.

In [17, the following result was obtained.

Theorem A. Let $p>p_{L}$. Suppose that $u_{0}$ is a radially symmetric function with $z\left(u_{0}-\varphi_{\infty}\right), z\left(\left(u_{0}\right)_{t}\right)<\infty$ and that $u$ is a solution of (1.4) which exhibits type II blow-up at $t=T$. Let $\xi_{i}(t)$ be the ith zero of $u(t)-\varphi_{\infty}$ for a positive integer $i$. Denote by $m$ the maximum over $i$ such that $\liminf _{t \nearrow T} \xi_{i}(t)=0$. Let $w$ be the solution 
of (1.8) corresponding to $u$. If $m$ is even, then there exist an integer $\ell \geq 2$ and a constant $c$ such that

$$
w(s)=\varphi_{\infty}+c e^{-\lambda_{\ell} s} \phi_{\ell}+o\left(e^{-\lambda_{\ell} s}\right) \quad \text { in } H_{\rho}^{1} \cap C_{l o c}^{1}(0, \infty) \text { as } s \rightarrow \infty .
$$

When we treat the problem (1.8), the standard theory of infinite dimensional dynamical system on refined asymptotics around a steady state cannot be applied even to solutions converging to a steady state $\kappa \equiv(p-1)^{-1 /(p-1)}$ as $s \rightarrow \infty$ in the subcritical case as mentioned in [1]. Therefore, refined asymptotic behavior such as 1.15) with $\varphi_{\infty}$ replaced by $\kappa$ was given by a series of estimates in 1], [5] and 18, which also contain the case of convergence slower than exponential rate. The singularity of $\varphi_{\infty}$ prevents us from applying their methods directly to solutions converging to $\varphi_{\infty}$ as $s \rightarrow \infty$. Thus the proof of Theorem A needed further estimates.

The following is the main result of [17, which is based on Theorem A.

Theorem B. Let $p>p_{L}$. Suppose that $u_{0}$ is a radially symmetric function with $z\left(u_{0}-\varphi_{\infty}\right), z\left(\left(u_{0}\right)_{t}\right)<\infty$. Let $u$ be a solution of (1.4) which undergoes type II blow-up at $t=T$. Let $\xi_{i}(t)$ be the ith zero of $u(t)-\varphi_{\infty}$ for a positive integer $i$. Denote by $m$ the maximum over $i$ such that $\liminf _{t \nearrow T} \xi_{i}(t)=0$. Let $w$ be the solution of (1.8) corresponding to $u$. When $m$ is even, let $\ell$ be the integer in Theorem $A$. Then there exist constants $C_{1}, C_{2}>0$ such that

$$
C_{1}(T-t)^{-\gamma_{\ell}} \leq|u(t)|_{\infty} \leq C_{2}(T-t)^{-\gamma_{\ell}} \quad \text { for } t \in[0, T),
$$

where

$$
\gamma_{j}=\frac{2 \lambda_{j}}{(p-1)|\alpha|-2} \quad \text { for } j=0,1,2, \ldots
$$

with the constant $\alpha$ in (1.12).

In the proof of Theorem B, intersections among three solutions play a crucial role. The intersection comparison argument between two solutions of a parabolic equation has possessed many applications in various problems. However it was not sufficient to prove Theorem B. She chose three suitable solutions $w_{1}, w_{2}, w_{3}$ of (1.8) and carefully watched the movement of intersections between $w_{1}(s)$ and $w_{2}(s), w_{2}(s)$ and $w_{3}(s), w_{3}(s)$ and $w_{1}(s)$. The method suggested a relation to the braid group theory. We apply the braid group theory to those three solutions used in [17.

Our purpose of the present paper is to show the following results according to the braid group theory. Here and hereafter, we number the zeros of function $f \neq \equiv$ in $[0, a)$ with $0<a \leq+\infty$ in order of size.

Theorem 1.1. Let $p>p_{J L}$ and $\lambda_{j} \neq 0$ for any $j \geq 2$. Suppose that $u_{0}$ is a radially symmetric function with $z\left(u_{0}-\varphi_{\infty}\right), z\left(\left(u_{0}\right)_{t}\right)<\infty$. Let $u$ be a solution of (1.4) or (1.5) which undergoes type II blow-up at $t=T$. Let $\xi_{i}(t)$ be the ith zero of $u(t)-\varphi_{\infty}$ for a positive integer $i$. Denote by $m$ the maximum over $i$ such that $\liminf _{t \nearrow T} \xi_{i}(t)=0$. Then there exists a constant $\bar{C}>0$ such that

$$
|u(t)|_{\infty} \leq \bar{C}(T-t)^{-\gamma_{m}-\frac{1}{p-1}} \quad \text { for } t \in[0, T),
$$

where $\gamma_{j}$ is the constant defined by (1.16). 
Theorem 1.2. Suppose that the same conditions as in Theorem 1.1 hold. If $m$ is even, then there exists $\underline{C}>0$ such that

$$
|u(t)|_{\infty} \geq \underline{C}(T-t)^{-\gamma_{m}-\frac{1}{p-1}} \quad \text { for } t \in[0, T) .
$$

Let $m$ be odd. Denote by $q$ the minimum over $j$ such that $\lambda_{j} \geq 0$. If $m \geq q+1$, then there exists a constant $C_{1}>0$ such that

$$
|u(t)|_{\infty} \geq C_{1}(T-t)^{-\gamma_{m-1}-\frac{1}{p-1}} \quad \text { for } t \in[0, T) .
$$

If for any $\varepsilon>0$ there exist $a, b>0$ and $t_{0} \geq 0$ such that

$$
\left|\frac{u(\xi, t)}{\varphi_{\infty}(\xi)}-1\right|<\varepsilon \quad \text { for } \xi \in\left[a(T-t)^{1 / 2}, b\right] \text { and } t \in\left[t_{0}, T\right),
$$

then (1.18) is valid with some constant $\underline{C}>0$.

Corollary 1.1. Under the hypothesis of Theorem 1.1, let $m$ be even. Then there exist constants $\underline{C}, \bar{C}>0$ such that

$$
\underline{C}(T-t)^{-\gamma_{m}-\frac{1}{p-1}} \leq|u(t)|_{\infty} \leq \bar{C}(T-t)^{-\gamma_{m}-\frac{1}{p-1}} \quad \text { for } t \in[0, T) .
$$

Remark 1.1. The blow-up rate $C(T-t)^{-\gamma_{m}-\frac{1}{p-1}}$ with some constant $C>0$ is that of a solution obtained in [6], [7] (see Proposition 2.3 below).

Remark 1.2. Let $\xi_{i}(t)$ be the $i$ th zero of $u(t)-\varphi_{\infty}$ for a positive integer $i$. In the process of the proof of Theorem B, it was shown that

$$
\liminf _{t \nearrow T} \xi_{1}(t) \geq C_{0}(T-t)^{1 / 2} \quad \text { for } t \in[0, T)
$$

with some constant $C_{0}>0$. Let $p>p_{L}$ in Corollary 1.1. According to Theorems A, B and Corollary 1.1, any $\xi_{i}(t)$ with $\liminf _{t \nearrow T} \xi_{i}(t)=0$ approaches $\xi=0$ at the backward selfsimilar rate, i.e., $\lim _{t \nearrow T}(T-t)^{-1 / 2} \xi_{i}(t)=C_{i}$ with some constant $C_{i}>0$.

The proofs of Theorems 1.1, 1.2 are simpler than that of Theorem B in [17 since we can avoid complicated analytic estimates by virtue of an application of braid group theory. Moreover the hypothesis on $p$ is improved to $p>p_{J L}$.

In order to show how the braid group theory is used, let us briefly explain the scenario to prove Theorem 1.1 in the case that $m$ is even, i.e., $m=2 k$ for a positive integer $k$. Let $w_{1}=w$ for a solution $w$ of (1.8) or (1.9) corresponding to a type II blow-up solution and $w_{2}=\varphi_{\infty}$. We assume that the conclusion does not hold. Then there exist a solution $w_{3}$ of (1.8),$d>0$ and $s_{1}, s_{2} \geq s^{T}$ such that

$$
w_{1}\left(d e^{s / 2}, s\right)<w_{3}\left(d e^{s / 2}, s\right)<w_{2}\left(d e^{s / 2}, s\right) \quad \text { for } s \in\left[s_{1}, s_{2}\right]
$$

and that

(i): at $s=s_{1}$ (Figure 1.1),

(i-1) $w_{1}\left(0, s_{1}\right)<w_{3}\left(0, s_{1}\right)$;

(i-2) for $\varepsilon, \delta>0$ sufficiently small,

$$
\sup _{r \in\left[\delta, d e^{s_{1} / 2}\right]}\left|w_{2}\left(r, s_{1}\right)-w_{3}\left(r, s_{1}\right)\right|<\varepsilon \sup _{r \in\left[\delta, d e^{s_{1} / 2}\right]}\left|w_{1}\left(r, s_{1}\right)-w_{2}\left(r, s_{1}\right)\right| ;
$$

(i-3) $w_{1}\left(s_{1}\right)-w_{3}\left(s_{1}\right)$ has $2 k$ zeros in $\left[0, d e^{s_{1} / 2}\right]$;

(ii): at $s=s_{2}$ (Figure 1.2),

(ii-1) $w_{1}\left(0, s_{2}\right)<w_{3}\left(0, s_{2}\right)$; 
(ii-2) all zeros of $w_{2}\left(s_{2}\right)-w_{3}\left(s_{2}\right)$ are closer to $r=0$ than the first zero of $w_{1}\left(s_{2}\right)-w_{2}\left(s_{2}\right)$

(ii-3) $w_{1}(0, s)-w_{3}(0, s)$ changes sign at least twice for $s \in\left(s_{1}, s_{2}\right)$.

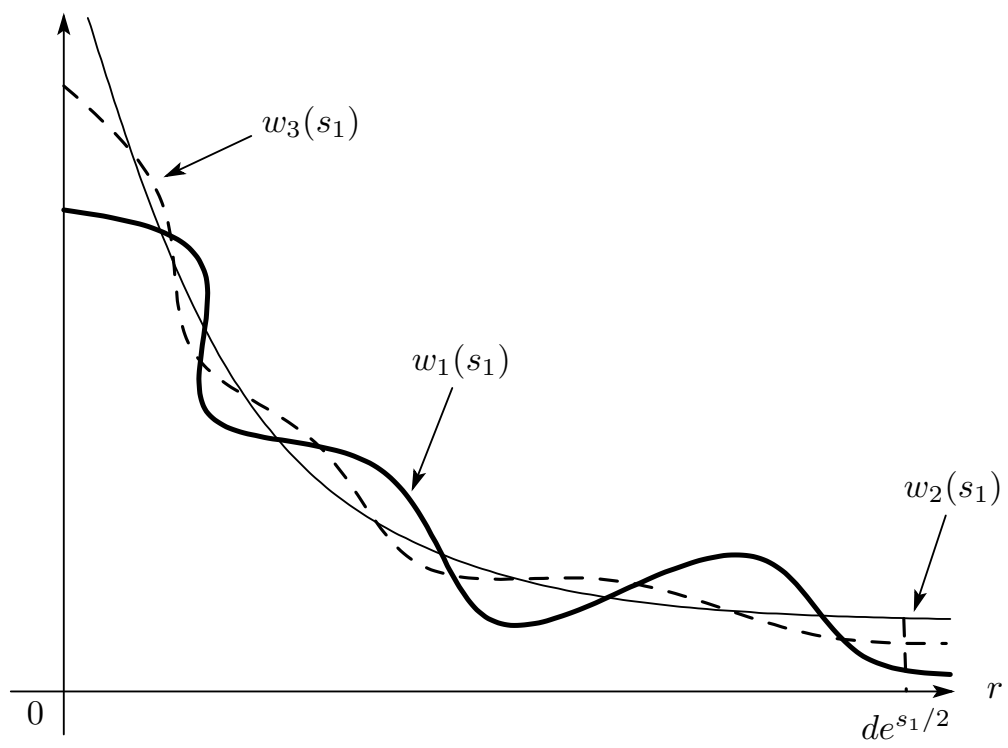

FiguRE 1.1

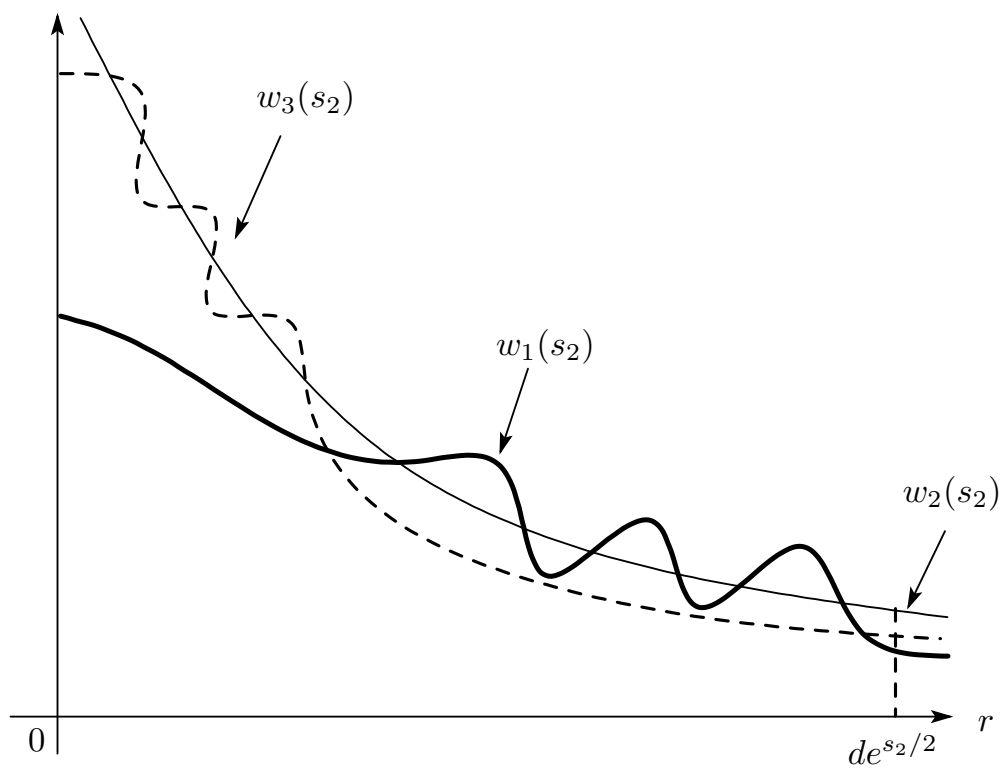

FIGURE 1.2 
Let $\mathbf{G}^{+}$be the semigroup of positive braids of three strands. Following $[2$ and [3], these three solutions are regarded as a positive braid. A braid constructed by the three solutions at $s=s_{1}$ is equivalent to $A_{2 k} \equiv\left(X Y^{2} X\right)^{k} Y^{2 k}$, where $X, Y$ are the generators. $B_{2 k} \equiv X^{2} Y^{2 k} X Y^{2 k} X$ is a parabolic reduction of a braid corresponding to the situation at $s=s_{2}$. Here the first $X^{2}$ in $B_{2 k}$ do not appear in Figure 1.2 since they represent intersections between $w_{1}(s)$ and $w_{2}(s)$ which disappear at $r=0$ for $s \in\left(s_{1}, s_{2}\right)$. The notion of parabolic reduction was introduced by [10]. For $A, B \in \mathbf{G}^{+}, B$ is called a simple parabolic reduction of $A$ if there exist $C, \widetilde{C}, D, \widetilde{D} \in \mathbf{G}^{+}$with $\widetilde{C} \sim C$ and $\widetilde{D} \sim D$ such that

$$
A \sim C X^{2} D, B \sim \widetilde{C} \widetilde{D} \quad \text { or } \quad A \sim C Y^{2} D, B \sim \widetilde{C} \widetilde{D} .
$$

Translating a simple parabolic reduction to a phenomenon in a parabolic equation, this means that two intersections between solutions disappear at some time. We say that $B$ is a parabolic reduction of $A$ if $A$ is deformed to $B$ by applying simple parabolic reductions finitely many times. In the above process, $B_{2 k}$ is a parabolic reduction of $A_{2 k}$. On the other hand, we prove that $B_{2 k}$ is not a parabolic reduction of $A_{2 k}$. This contradiction implies that Theorem 1.1 is valid.

In [2] and [3], the dynamics on braids are studied and applied to the qualitative investigation of partial differential equations. Their point of view is different from ours. The braid invariants of periodic solutions to an ordinary differential equation were studied in [13]. He treated an ordinary differential equation and investigated the braid invariants. On the other hand, we are concerned with a partial differential equation and the parabolic reduction. A summary of related results to the present paper was published in the overview paper [9].

The present paper is organized as follows: In Section 2, we prepare analytic estimates. In Section 3, we review fundamental facts on the braid group, and obtain lemmas on the parabolic reduction which play an essential role in our proof of Theorems 1.1, 1.2. Section 4 is devoted to the proof of the main theorems. In Section 5, we show that Lemma 3.3 obtained in Section 3 is optimal.

\section{Analytic preparation}

We begin this section by a result given in [17.

Proposition 2.1. When $p>p_{J L}$, let a radially symmetric function $u_{0}$ satisfy $z\left(u_{0}-\varphi_{\infty}\right), z\left(\left(u_{0}\right)_{t}\right)<\infty$. If a solution $u$ of (1.4) or (1.5) with initial data $u_{0}$ exhibits type II blow-up at $t=T$, then $\liminf _{t \nearrow T} \frac{u(0, t)}{|u(t)|_{\infty}}>0$.

The following was shown in [12.

Proposition 2.2. When $p>p_{J L}$, let $q$ be the minimum over $j$ such that $\lambda_{j} \geq 0$. Let $u$ be a solution of (1.4) with $z\left(u_{0}-\varphi_{\infty}\right)<\infty$ or (1.5) which exhibits type II blow-up at $t=T$. Denote by $\xi_{i}(t)$ the ith zero of $u(t)-\varphi_{\infty}$ for a positive integer $i$. Let $m$ be the maximum over $i$ such that $\liminf _{t \nearrow T} \xi_{i}(t)=0$. Then it follows that $m \geq q$.

For $a>0$, let $\varphi_{a}$ be the solution of

$$
\left\{\begin{array}{l}
\varphi^{\prime \prime}+\frac{N-1}{\xi} \varphi^{\prime}+\varphi^{p}=0 \quad \text { in }(0, \infty), \\
\varphi(0)=a, \quad \varphi^{\prime}(0)=0 .
\end{array}\right.
$$


It was shown in [8] that if $p>p_{J L}$, then $\varphi_{a}$ is increasing with respect to $a$ and

$$
\varphi_{a}(\xi)=\varphi_{\infty}(\xi)-k(a) \xi^{-|\alpha|}+o\left(\xi^{-|\alpha|}\right) \quad \text { as } \xi \rightarrow \infty
$$

for some $k(a)>0$, where $\alpha$ is the constant in (1.12). The following was given in [6], 7] (also see [15]).

Proposition 2.3. Let $p>p_{J L}$. Then for any nonnegative integer $\ell$ with $\lambda_{\ell}>0$ there exists a solution $w_{\ell}$ of (1.8) with initial data $w_{\ell}\left(s^{T}\right) \in L^{\infty}$ having $\ell$ intersections with $\varphi_{\infty}$ which satisfies the following for some constants $K>0,0<\sigma<1 / 2$ :

(i) Let $\gamma_{j}$ be the constant in (1.16) and $\eta_{j}=(p-1) \gamma_{j} / 2$. Let $c_{\ell}$ be the constant in (1.13) with $j=\ell$ and $\underline{\varphi}_{\ell}, \bar{\varphi}_{\ell}$ be the solutions of (2.1) with $k(a)=\underline{k}_{\ell}, \bar{k}_{\ell}$ in (2.2) for $\underline{k}_{\ell}, \bar{k}_{\ell}$ with $\underline{k}_{\ell}>c_{\ell}>\bar{k}_{\ell}$, respectively. Then it follows that

$$
e^{\gamma_{\ell} s} \underline{\varphi}_{\ell}\left(e^{\eta_{\ell} s} r\right)<w_{\ell}(r, s)<e^{\gamma_{\ell} s} \bar{\varphi}_{\ell}\left(e^{\eta_{\ell} s} r\right)
$$

for $r \in\left[0, K e^{-\eta_{\ell} s}\right]$ and $s \geq s^{T}$.

(ii) For sufficiently small $\varepsilon>0$, it follows that

$$
\begin{aligned}
& \left|w_{\ell}(r, s)-\left\{\varphi_{\infty}(r)-e^{-\lambda_{\ell} s} \phi_{\ell}(r)\right\}\right| \leq \varepsilon e^{-\lambda_{\ell} s}\left(r^{-|\alpha|}+r^{2 \lambda_{\ell}-\frac{2}{p-1}}\right) \\
& \text { for } r \in\left[K e^{-\eta_{\ell} s}, e^{\sigma s}\right] \text { and } s \geq s^{T} .
\end{aligned}
$$

In the situation of Proposition 2.2, there exists a solution $w_{m}$ of (1.8) obtained in Proposition 2.3 with $\ell=m$ if $\lambda_{j} \neq 0$ for all $j \geq 0$.

Remark 2.1. By the choice of initial data $w\left(s^{T}\right)$ in the proof of Proposition 2.3, it follows that

$$
\left|w\left(r, s^{T}\right)-\varphi_{\infty}(r)\right| \leq e^{-c s^{T}} \varphi_{\infty}(r) \quad \text { for } r \in\left[\widetilde{K} e^{-\eta_{\ell} s^{T}}, e^{\widetilde{\sigma} s^{T}}\right]
$$

with some constants $c>0,0<\widetilde{K}<K$ and $\sigma<\widetilde{\sigma}<1 / 2$. On the other hand, the condition of $w\left(s^{T}\right)$ in $\left(e^{\widetilde{\sigma} s^{T}}, \infty\right)$ is only that $w\left(r, s^{T}\right) \leq C r^{-2 /(p-1)}$ for $r>e^{\widetilde{\sigma} s^{T}}$ with some constant $C>0$.

Lemma 2.1. When $p>p_{J L}$, let $\ell$ be an integer with $\lambda_{\ell}>0$. Then for sufficiently large $a>0$ and sufficiently small $\varepsilon, b, T>0$ there exists a solution $w_{\ell}$ of (1.8) obtained in Proposition 2.3 such that

$$
(1-\varepsilon) \varphi_{\infty}(r)<w_{\ell}(r, s)<(1+\varepsilon) \varphi_{\infty}(r)
$$

for $r \in\left[a, b e^{s / 2}\right]$ and $s \geq s^{T}$.

Proof. Let $w_{\ell}$ be a solution of (1.8) such that

$$
W_{\ell}\left(r, s^{T}\right)= \begin{cases}W_{\ell}\left(e^{\widetilde{\sigma} s^{T}}, s^{T}\right)\left\{1-\left(r-e^{\widetilde{\sigma} s^{T}}\right)\right\} & \text { for } r \in\left[e^{\widetilde{\sigma} s^{T}}, e^{\widetilde{\sigma} s^{T}}+1\right], \\ 0 & \text { for } r \in\left[e^{\widetilde{\sigma} s^{T}}+1, \infty\right),\end{cases}
$$

where $W_{\ell}(r, s)=w_{\ell}(r, s)-\varphi_{\infty}(r)$ for $r>0$ and $s \geq s^{T}$ and $\widetilde{\sigma}$ is the constant in (2.3). We denote $w_{\ell}$ and $W_{\ell}$ by $w$ and $W$, respectively, for simplicity. Since (2.4) is immediate for $r \in\left[a, e^{\sigma s}\right]$ and $s \geq s^{T}$ if $T$ is sufficiently small, by Proposition 2.3. it suffices to show it for $r \in\left[e^{\sigma s}, b e^{s / 2}\right]$ and $s \geq s^{T}$, where $\sigma$ is the constant in Proposition 2.3 .

In order to prove the first inequality in (2.4), we suppose that $\ell$ is even since it is trivial if $\ell$ is odd. For $\underline{c}>0$ determined later, put

$$
\underline{W}(r, s)=\underline{c} e^{-\lambda_{\ell} s} \phi_{\ell}(r) \quad \text { for } r>0 \text { and } s \geq s^{T}
$$


and

$$
\underline{w}(r, s)=\varphi_{\infty}(r)+\underline{W}(r, s) \quad \text { for } r>0 \text { and } s \geq s^{T} .
$$

We easily see that $\underline{w}$ is a subsolution of (1.8). Denote by $R_{i}$ the $i$ th zero of $\phi_{\ell}$ for $i=1,2, \ldots, \ell$. By the choice of $W\left(s^{T}\right)$, there exist $c_{0}, \delta>0$ such that for $\underline{c} \geq c_{0}$,

$$
w\left(r, s^{T}\right) \geq \underline{w}\left(r, s^{T}\right) \quad \text { for } r \geq R_{\ell}+\delta .
$$

Let $\underline{r}(s)$ be the zero of $w(s)-\underline{w}(s)$ such that $\underline{r}\left(s^{T}\right)$ is the nearest to $R_{\ell}+\delta$. From Proposition 2.3, there exists $c_{1} \geq c_{0}$ such that for $\underline{c} \geq c_{1}$,

$$
w\left(e^{\sigma s}, s\right)>\underline{w}\left(e^{\sigma s}, s\right) \quad \text { for } s \geq s^{T}
$$

and hence

$$
\underline{r}(s)<e^{\sigma s} \quad \text { for } s \geq s^{T} .
$$

By the comparison theorem, we see that

$$
w(r, s) \geq \underline{w}(r, s) \quad \text { for } r \geq \underline{r}(s) \text { and } s \geq s^{T} .
$$

It follows from (1.14) that

$$
e^{-\lambda_{\ell} s}\left|\phi_{\ell}(r)\right| \leq 2\left|\widetilde{c}_{\ell}\right| b^{2 \lambda_{\ell}} r^{-\frac{2}{p-1}} \quad \text { for } r \in\left[e^{\sigma s}, b e^{s / 2}\right] \text { and } s \geq s^{T},
$$

where $\widetilde{c}_{\ell}$ is the constant in (1.14) with $j=\ell$. This implies that

$$
\underline{w}(r, s)>\varphi_{\infty}(r)-\varepsilon \varphi_{\infty}(r) \quad \text { for } r \in\left[e^{\sigma s}, b e^{s / 2}\right] \text { and } s \geq s^{T}
$$

if we take $b>0$ with $2 \underline{c}\left|\widetilde{c}_{\ell}\right| b^{2 \lambda_{\ell}}<\varepsilon c_{\infty}$. Thus the first inequality in (2.4) is valid for $r \in\left[e^{\sigma s}, b e^{s / 2}\right]$ and $s \geq s^{T}$ from (2.5)-2.7).

In order to prove the second inequality of (2.4), we suppose that $\ell$ is odd since it is trivial if $\ell$ is even. By the choice of $W\left(s^{T}\right)$, we have

$$
w\left(r, s^{T}\right) \leq\left(1+\frac{\varepsilon}{4}\right) \varphi_{\infty}(r) \quad \text { for } r>0
$$

for sufficiently small $T>0$. Put

$$
\bar{s}=\sup \left\{s>s^{T}: w(r, \tau)<(1+2 \varepsilon) \varphi_{\infty}(r) \text { for } r>0 \text { and } \tau \in\left[s^{T}, s\right)\right\}
$$

and

$$
s^{*}=\sup \left\{s>s^{T}: w(r, \tau)<(1+\varepsilon) \varphi_{\infty}(r) \text { for } r>0 \text { and } \tau \in\left[s^{T}, s\right)\right\} .
$$

Assume that $s^{*}<\infty$. Put $\bar{t}=T-e^{-\bar{s}}$ and $t^{*}=T-e^{-s^{*}}$. Let $u$ be a solution of (1.4) corresponding to $w$. The solution $u$ is written as

$$
\begin{aligned}
u(x, t)= & \frac{1}{(4 \pi t)^{N / 2}} \int_{\mathbf{R}^{N}} u_{0}(y) \exp \left(-\frac{|x-y|^{2}}{4 t}\right) d y \\
& +\int_{0}^{t} \frac{1}{(4 \pi(t-s))^{N / 2}} \int_{\mathbf{R}^{N}} u(y, s)^{p} \exp \left(-\frac{|x-y|^{2}}{4(t-s)}\right) d y d s
\end{aligned}
$$

for $x \in \mathbf{R}^{N}$ and $t \in(0, T)$.

Let $\psi_{1}(x)=|x|^{-\frac{2}{p-1}}$ and $\psi_{2}(x)=|x|^{-\frac{2 p}{p-1}}$ for $x \in \mathbf{R}^{N}$. It is immediate that $\psi_{1}$ and $\psi_{2}$ are supersolutions of the heat equation in $\mathbf{R}^{N}$ if $p>p_{J L}$. It follows from (2.8) that

$$
\begin{aligned}
u(x, t) & \leq c_{\infty}\left(1+\frac{\varepsilon}{4}\right)|x|^{-\frac{2}{p-1}}+\int_{0}^{t} c_{\infty}^{p}(1+2 \varepsilon)^{p}|x|^{-\frac{2 p}{p-1}} d s \\
& =c_{\infty}\left(1+\frac{\varepsilon}{4}\right)|x|^{-\frac{2}{p-1}}+c_{\infty}^{p}(1+2 \varepsilon)^{p} t|x|^{-2}|x|^{-\frac{2}{p-1}}
\end{aligned}
$$


for $t \in[0, \bar{t}]$. Suppose now that $|x|>b$ and $t \in[0, \bar{t}]$. Then we get

$$
c_{\infty}^{p}(1+2 \varepsilon)^{p} t|x|^{-2} \leq c_{\infty}^{p}(1+2 \varepsilon)^{p} b^{-2} \bar{t} .
$$

Let $T>0$ satisfy

$$
c_{\infty}^{p}(1+2 \varepsilon)^{p} b^{-2} T<\frac{\varepsilon}{4} c_{\infty} .
$$

Then it follows that

$$
u(x, t)<c_{\infty}\left(1+\frac{\varepsilon}{2}\right)|x|^{-\frac{2}{p-1}} .
$$

Therefore there exists $r^{*} \in\left(e^{\sigma s^{*}}, b e^{s^{*} / 2}\right]$ such that

$$
w\left(r^{*}, s^{*}\right)=(1+\varepsilon) \varphi_{\infty}\left(r^{*}\right) .
$$

Denote by $r_{i}(s)$ be the $i$ th zero of $w(s)-\varphi_{\infty}$ for $i=1,2, \ldots, \ell$. Since $W(r, s)>0$ for $r>r_{\ell}(s)$ and $s \geq s^{T}$, we have

$$
W_{s} \leq W_{r r}+\frac{N-1}{r} W_{r}-\frac{r}{2} W_{r}-\frac{1}{p-1} W+\frac{p c_{\infty}^{p-1}(1+\varepsilon)^{p-1}}{r^{2}} W
$$

for $r \geq r_{\ell}(s)$ for $s \in\left[s^{T}, s^{*}\right]$. We now consider an eigenvalue problem

$$
\phi^{\prime \prime}+\frac{N-1}{r} \phi^{\prime}-\frac{r}{2} \phi^{\prime}-\frac{1}{p-1} \phi+\frac{p c_{\infty}^{p-1}(1+\varepsilon)^{p-1}}{r^{2}} \phi=-\lambda \phi \quad \text { in } H_{\rho}^{1} .
$$

The following was shown in [14]: Let $p>p_{J L}$. Then the spectrum of (2.11) consists of countable eigenvalues for sufficiently small $\varepsilon>0$, and the $j$ th eigenvalue $\lambda_{j}^{\varepsilon}$ was given by

$$
\lambda_{j}^{\varepsilon}=\frac{\alpha_{\varepsilon}}{2}+\frac{1}{p-1}+j \quad \text { for } j=0,1,2, \ldots
$$

where

$$
\alpha_{\varepsilon}=\frac{-(N-2)+\sqrt{(N-2)^{2}-4 p c_{\infty}^{p-1}(1+\varepsilon)^{p-1}}}{2} .
$$

Furthermore the $j$ th eigenfunction $\phi_{j}^{\varepsilon}$ normalized in $H_{\rho}^{1}$ which is positive near $r=0$ fulfills (1.14) with $\lambda_{j}$ and $\widetilde{c}_{j}$ replaced by $\lambda_{j}^{\varepsilon}$ and some constant $\widetilde{c}_{j}^{\varepsilon}$. Setting $\bar{W}(r, s)=\bar{c} e^{-\lambda_{\ell}^{\varepsilon} s} \phi_{\ell}^{\varepsilon}(r)$ for $r>0$ and $s \geq s^{T}$ with a constant $\bar{c}>0$ to be determined later, $\bar{W}$ satisfies

$$
W_{s}=W_{r r}+\frac{N-1}{r} W_{r}-\frac{r}{2} W_{r}-\frac{1}{p-1} W+\frac{p c_{\infty}^{p-1}(1+\varepsilon)^{p-1}}{r^{2}} W .
$$

Put $\bar{w}(r, s)=\varphi_{\infty}(r)+\bar{W}(r, s)$. Taking $\bar{c}>0$ sufficiently large and $b>0$ with $4 \bar{c}\left|\widetilde{c}_{\ell}^{\varepsilon}\right| b^{2 \lambda_{\ell}^{\varepsilon}}<\varepsilon c_{\infty}$ for sufficiently small $\varepsilon>0$, we have

$$
\bar{w}(r, s) \leq \varphi_{\infty}(r)+\frac{\varepsilon}{2} \varphi_{\infty}(r) \quad \text { for } r \in\left[e^{\sigma s}, b e^{s / 2}\right] \text { and } s \in\left[s^{T}, s^{*}\right]
$$

in the same way as the proof of (2.7). Thus it follows from (2.10) and (2.12) that

$$
w(r, s)<\varphi_{\infty}(r)+\frac{\varepsilon}{2} \varphi_{\infty}(r) \quad \text { for } r \in\left[e^{\sigma s}, b e^{s / 2}\right] \text { and } s \in\left[s^{T}, s^{*}\right]
$$

by the comparison theorem, which contradicts (2.9). This completes the proof of the second inequality in (2.4). 
We recall a solution introduced in [17. For $\widetilde{T}>0$, let $W$ be a solution of (1.8) or (1.9) with $T$ replaced by $\widetilde{T}$. Define $\widetilde{W}$ by

$$
\begin{aligned}
& \widetilde{W}(r, s) \\
& =e^{-\frac{s}{p-1}}\left(\widetilde{T}-T+e^{-s}\right)^{-\frac{1}{p-1}} W\left(e^{-s / 2}\left(\widetilde{T}-T+e^{-s}\right)^{-1 / 2} r,-\log \left(\widetilde{T}-T+e^{-s}\right)\right)
\end{aligned}
$$

for $r \in[0, \infty)$ and $s \in\left[s^{T}, \widetilde{S}\right)$, where $\widetilde{S}=-\log (T-\widetilde{T})$ if $\widetilde{T}<T$ and $\widetilde{S}=\infty$ if $\widetilde{T} \geq T$. Then $\widetilde{W}$ is a solution of (1.8) or (1.9) which blows up at $s=\widetilde{S}$ if $\widetilde{T}<T$. As in [17, $\widetilde{W}$ and $\widetilde{W}(\cdot+\tau)$ with $\tau \in \mathbf{R}$ play an important role in this paper.

The following result was given in [17].

Lemma 2.2. Let $p>p_{J L}$. If $u$ is a solution of (1.4) or (1.5) with $z\left(u_{0}-\varphi_{\infty}\right)<$ $+\infty$, then $u(T) \not \equiv \varphi_{\infty}$, where $u(T)$ is the blow-up profile defined by $u(\xi, T)=$ $\lim _{t \succ T} u(\xi, t)$ for $\xi>0$.

Lemma 2.3. Let $p>p_{J L}$ and $\lambda_{j} \neq 0$ for any $j \geq 2$. Suppose that $u_{0}$ is a radially symmetric function with $z\left(u_{0}-\varphi_{\infty}\right), z\left(\left(u_{0}\right)_{t}\right)<\infty$. Let $u$ be a solution of (1.4) or (1.5) which undergoes type II blow-up at $t=T$. Let $\xi_{i}(t)$ be the ith zero of $u(t)-\varphi_{\infty}$ for a positive integer $i$, and denote by $m$ the maximum over $i$ such that $\liminf _{t \nearrow T} \xi_{i}(t)=0$. Let $w$ be a solution of (1.8) or (1.9) corresponding to $u$. Let $w_{m}$ be a solution of (1.8) with $\ell=m$ obtained in Proposition 2.3 with $T$ replaced by $0<\widetilde{T}<T$, and let $\widetilde{w}_{m}$ be a solution defined in (2.13) with $W=w_{m}$. Then there exist $s_{0} \geq s^{T}, C>0$ and $d>0$ such that for $\tau \geq 2 \log d+C$,

(i) $w(s)-\varphi_{\infty}$ has at most $m$ zeros in $\left[0, d e^{s / 2}\right)$ for $s \geq s_{0}$;

(ii) $w\left(d e^{s / 2}, s\right)<\widetilde{w}_{m}\left(d e^{s / 2}, s+\tau\right)$ for $s \in\left[s_{0}, \widetilde{S}-\tau\right)$ if $m$ is even, and $w\left(d e^{s / 2}, s\right)>\widetilde{w}_{m}\left(d e^{s / 2}, s+\tau\right)$ for $s \in\left[s_{0}, \widetilde{S}-\tau\right)$ if $m$ is odd, where $\widetilde{S}=-\log (T-\widetilde{T})$.

Proof. By Lemma 2.2, there exist $d>0, s_{0} \geq s^{T}$ and $\mu \in(0,1)$ such that

(i) $w(s)-\varphi_{\infty}$ has $m$ zeros in $\left[0, d e^{s / 2}\right)$ for $s \in\left[s_{0}, \infty\right)$;

(ii) if $m$ is even, then $w\left(d e^{s / 2}, s\right)<\mu \varphi_{\infty}\left(d e^{s / 2}\right)$ for $s \geq s_{0}$;

(iii) if $m$ is odd, then $w\left(d e^{s / 2}, s\right)<\frac{1}{\mu} \varphi_{\infty}\left(d e^{s / 2}\right)$ for $s \geq s_{0}$.

Suppose that $m$ is even. According to Lemma 2.1 for $0<\varepsilon<1-\mu$ and sufficiently large $a>0$ and small $b>0$ there exists $\widetilde{s}_{0} \geq s^{\widetilde{T}}$ such that

$$
w_{m}(\widetilde{r}, \widetilde{s})>(1-\varepsilon) \varphi_{\infty}(\widetilde{r}) \quad \text { for } \widetilde{r} \in\left[a, b e^{\widetilde{s} / 2}\right] \text { and } \widetilde{s} \geq \widetilde{s}_{0} .
$$

Let $\tau>2 \log (d / b)$. Put $s_{1}=\max \left\{s_{0},-\log \left(T-\widetilde{T}+e^{-\widetilde{s}_{0}}\right)-\tau\right\}$. By the definition of $\widetilde{w}_{m}$, if

$$
a \leq e^{-\frac{s+\tau}{2}}\left(\widetilde{T}-T+e^{-(s+\tau)}\right)^{-1 / 2} r \leq b e^{\widetilde{s} / 2}
$$

with $\widetilde{s}=-\log \left(\widetilde{T}-T+e^{-(s+\tau)}\right)$, then

$$
\widetilde{w}_{m}(r, s+\tau)>(1-\varepsilon) \varphi_{\infty}(r) \quad \text { for } s \in\left[s_{1}, \widetilde{S}-\tau\right) .
$$

By the choice of $\tau$, (2.14) is valid for $r=d e^{s / 2}$ and $s \in\left[s_{1}, \widetilde{S}-\tau\right)$. Therefore we have

$$
\widetilde{w}_{m}\left(d e^{s / 2}, s+\tau\right)>(1-\varepsilon) \varphi_{\infty}\left(d e^{s / 2}\right) \quad \text { for } s \in\left[s_{1}, \widetilde{S}-\tau\right) .
$$


It follows from (ii) and (2.15) that

$$
\widetilde{w}_{m}\left(d e^{s / 2}, s+\tau\right)>w\left(d e^{s / 2}, s\right) \quad \text { for } s \in\left[s_{1}, \widetilde{S}-\tau\right) .
$$

The proof in the case that $m$ is odd is similar. This completes the proof.

\section{ON THE BRAID GROUP}

We first review the fundamental results on braid group theory. Let $\mathbf{G}$ be the braid group of three strands. Denote by $X, Y$ the generators of $\mathbf{G}$ as in the following figure:
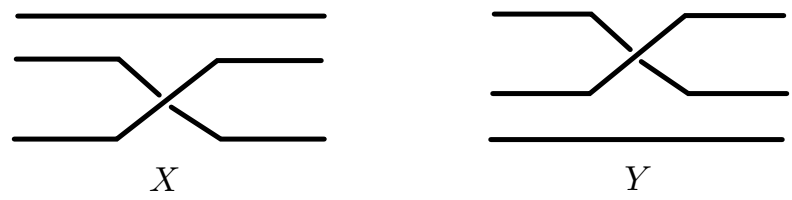

and by $I$ the trivial braid in $\mathbf{G}$ shown below:

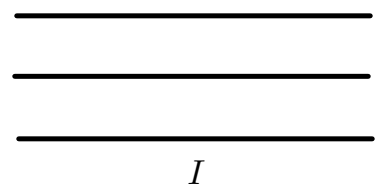

It is well known that Artin's formula

$$
X Y X=Y X Y
$$

is valid. It follows from (3.1) that

$$
\begin{aligned}
& X Y^{2} X Y^{2}=Y X^{2} Y X^{2}=X Y X^{2} Y X=Y X Y^{2} X Y \\
& \quad=X^{2} Y X^{2} Y=Y^{2} X Y^{2} X=X Y X Y X Y=Y X Y X Y X .
\end{aligned}
$$

We also get

$$
Y^{2 k} X Y=X Y X^{2 k} \quad \text { and } \quad X^{2 k} Y X=Y X Y^{2 k}
$$

for each positive integer $k$. In fact, it is immediate from (3.1) in the case of $k=1$. Suppose that (3.3) with $k$ holds. Then

$$
Y^{2(k+1)} X Y=Y^{2} Y^{2 k} X Y=Y^{2} X Y X^{2 k}=X Y X^{2} X^{2 k}=X Y X^{2(k+1)} \text {. }
$$

This implies that $Y^{2 k} X Y=X Y X^{2 k}$ for any positive integer $k$. The same argument yields the second equality in (3.3) for each positive integer $k$.

For $A, B \in \mathbf{G}$, we say that $A$ is equivalent to $B$, i.e., $A \sim B$ if $A$ is modified to $B$ by applying (3.1) at most finitely many times. In other words, $A \sim B$ iff $A$ is topologically equivalent to $B . A \in \mathbf{G}$ is called a positive braid if it contains neither $X^{-1}$ nor $Y^{-1}$. Denote by $\mathbf{G}^{+}$the semigroup of positive braids in $\mathbf{G}$. For $A \in \mathbf{G}^{+}$, denote by $\ell(A)$ the number of intersections included in $A$. If $A \sim B$ for $A, B \in \mathbf{G}^{+}$, then $\ell(A)=\ell(B)([3])$.

Let $\mathcal{X}, \mathcal{Y}$ be the following two matrices:

$$
\mathcal{X}=\left(\begin{array}{rr}
-t & t \\
0 & 1
\end{array}\right), \quad \mathcal{Y}=\left(\begin{array}{rr}
1 & 0 \\
1 & -t
\end{array}\right)
$$

with $t \in \mathbf{R}$. When $A \in \mathbf{G}$ is written as

$$
A=X^{i_{1}} Y^{j_{1}} X^{i_{2}} Y^{j_{2}} \cdots X^{i_{n}} Y^{j_{n}}
$$


for integers $i_{k}, j_{k}$ for $k=1,2, \ldots, n$, let

$$
\mathcal{A}=\mathcal{X}^{i_{1}} \mathcal{Y}^{j_{1}} \mathcal{X}^{i_{2}} \mathcal{Y}^{j_{2}} \ldots \mathcal{X}^{i_{n}} \mathcal{Y}^{j_{n}} .
$$

Then $\mathcal{A}$ is called the Burau representation of $A$. If $A \sim B$ for $A, B \in \mathbf{G}$, then $\mathcal{A}=\mathcal{B}$ for all $t$ for their Burau representations $\mathcal{A}, \mathcal{B}$ (even for braid groups with $k$ strands with $k \geq 3$ ). Moreover the converse statement is valid for the braid group of three strands. In the rest of this paper, we denote by $A$ the Burau representation $\mathcal{A}$ of $A \in \mathbf{G}$ for simplicity. Since $X Y X Y X Y=t^{3} I$ with the unit matrix $I$ by easy calculation, we see that

$$
\begin{aligned}
& X Y^{2} X Y^{2}=Y X^{2} Y X^{2}=X Y X^{2} Y X=Y X Y^{2} X Y \\
& \quad=X^{2} Y X^{2} Y=Y^{2} X Y^{2} X=X Y X Y X Y=Y X Y X Y X=t^{3} I
\end{aligned}
$$

from (3.1).

We next state the definition of parabolic reduction introduced in [10]. Let $A, B \in$ $\mathbf{G}^{+}$. We say that $B$ is a simple parabolic reduction of $A$ if there exist $C, \widetilde{C}, D, \widetilde{D} \in$ $\mathbf{G}^{+}$with $\widetilde{C} \sim C$ and $\widetilde{D} \sim D$ such that

$$
A \sim C X^{2} D, B \sim \widetilde{C} \widetilde{D} \quad \text { or } \quad A \sim C Y^{2} D, B \sim \widetilde{C} \widetilde{D},
$$

and denote by $A \Rightarrow_{1} B$. If there exist $A_{1}, A_{2}, \ldots, A_{k} \in \mathbf{G}^{+}$with some positive integer $k$ such that $A \Rightarrow_{1} A_{1} \Rightarrow_{1} A_{2} \Rightarrow_{1} \cdots \Rightarrow_{1} A_{k} \Rightarrow_{1} B$, then $B$ is called a parabolic reduction of $A$, and it is denoted by $A \Rightarrow B$.

Following [2] and [3], let $a(s), b(s) \in \mathbf{R}$ with $a(s)<b(s)$ for $s \in\left[S_{1}, S_{2}\right]$. Let $v_{1}, v_{2}, v_{3}$ be solutions of a parabolic equation

$$
v_{s}=\alpha(r) v_{r r}+\beta(r) v_{r}+f(r, v) \quad \text { in }(a(s), b(s)) \text { for } s \in\left(S_{1}, S_{2}\right) .
$$

Here $\alpha, \beta, f$ are smooth and $\alpha$ is positive for $r \in[a(s), b(s)]$ and $s \in\left[S_{1}, S_{2}\right]$. (See Figure 3.1).

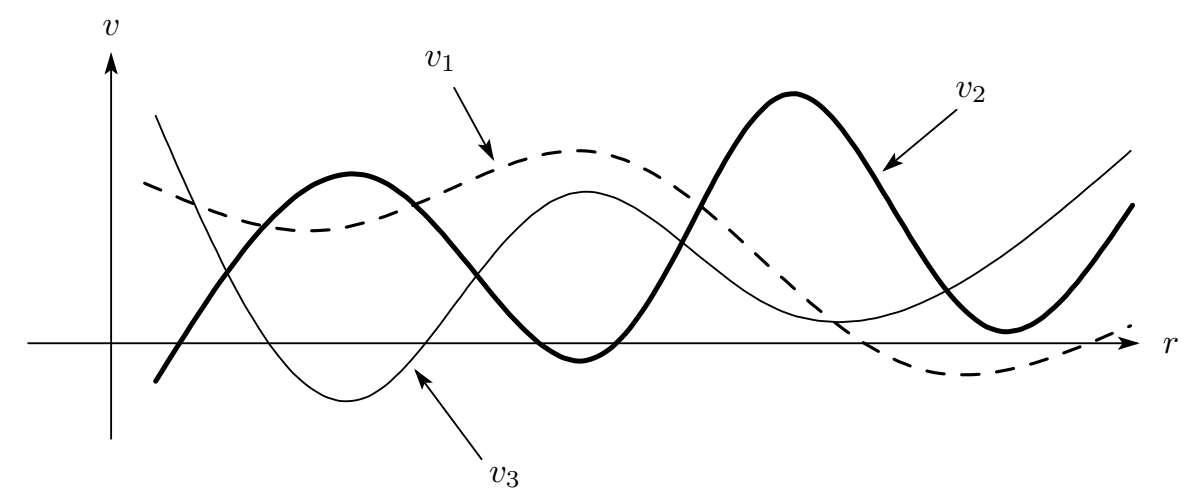

FIGURE 3.1

When $v_{i}(s)$ and $v_{j}(s)$ with $i \neq j$ transversally intersect at each of their intersections, that is, any zero of $v_{i}(s)-v_{j}(s)$ is nondegenerate, let us consider these three solutions $v_{1}, v_{2}, v_{3}$ in the space $\left\{\left(\partial_{r} v, r, v\right): r \in[a(s), b(s)]\right\}$. (See Figure 3.2).

Then $\left(v_{1}, v_{2}, v_{3}\right)$ can be regarded as an element of $\mathbf{G}^{+}$as in Figure 3.3. We get an abstract result. 


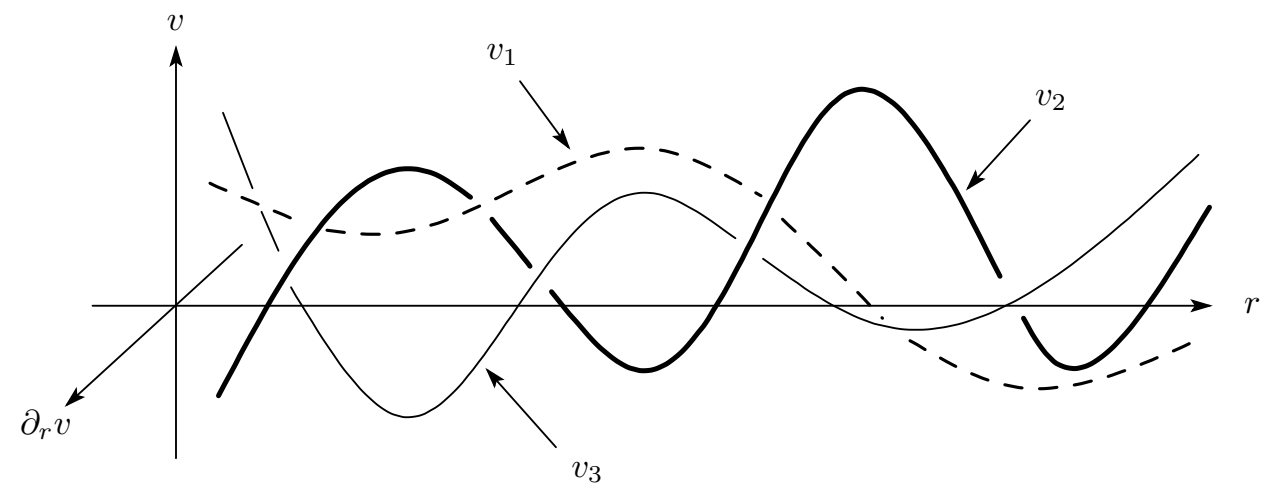

FiguRE 3.2

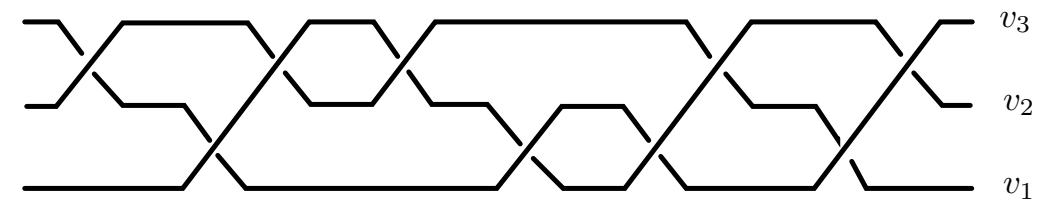

FiguRE 3.3

Lemma 3.1. Let $A, B, H \in \mathbf{G}^{+}$. If $H A \Rightarrow H B$, then $A \Rightarrow B$. If $A H \Rightarrow B H$, then $A \Rightarrow B$.

Proof. We first note that for $A \in \mathbf{G}^{+}$there exists $\widetilde{A} \in \mathbf{G}^{+}$such that $A X Y X=$ $X Y X \widetilde{A}$. Indeed, if $\ell(A)=1$, then the assertion is trivial from (3.1). Suppose that the assertion is valid if $\ell(A) \leq k$. Let $A \in \mathbf{G}^{+}$with $\ell(A)=k+1$. If $A=X A^{*}$ with $A^{*} \in \mathbf{G}^{+}$, then $A X Y X=X A^{*} X Y X=X X Y X \widetilde{A}^{*}=X Y X Y \widetilde{A}^{*}$ for some $\widetilde{A}^{*} \in \mathbf{G}^{+}$. If $A=Y A_{*}$ with $A_{*} \in \mathbf{G}^{+}$, then $A X Y X=Y A_{*} X Y X=Y X Y X \widetilde{A}_{*}=$ $X Y X X \widetilde{A}_{*}$ for some $\widetilde{A}_{*} \in \mathbf{G}^{+}$. Therefore the assertion holds for any $A \in \mathbf{G}^{+}$.

Let $\boldsymbol{\Lambda}=\left\{A \in \mathbf{G}^{+}: A\right.$ contains $\left.X Y X\right\}$. We prove that

$$
\left\{\begin{array}{l}
X A \Rightarrow_{1} X B \quad \Rightarrow \quad A \Rightarrow_{1} B \\
Y A \Rightarrow_{1} Y B \quad \Rightarrow \quad A \Rightarrow_{1} B
\end{array}\right.
$$

It is immediate if $\ell(B)=1$. Suppose that (3.6) holds if $\ell(B) \leq k$. Let $\ell(B)=k+1$ and $X A \Rightarrow_{1} X B$. Then there exist $C, \widetilde{C}, D, \widetilde{D} \in \mathbf{G}^{+}$with $C \sim \widetilde{C}$ and $D \sim \widetilde{D}$ such that

$$
X A=C X^{2} D, X B=\widetilde{C} \widetilde{D} \quad \text { or } \quad X A=C Y^{2} D, X B=\widetilde{C} \widetilde{D} .
$$

The following cases are possible:

(i) $C=X C_{1}, \widetilde{C}=X \widetilde{C}_{1}$ with $C_{1}, \widetilde{C}_{1} \in \mathbf{G}^{+}$;

(ii) $C=X C_{2}, \widetilde{C}=Y \widetilde{C}_{2}$ with $C_{2}, \widetilde{C}_{2} \in \mathbf{G}^{+}$;

(iii) $C=Y C_{3}, \widetilde{C}=X \widetilde{C}_{3}$ with $C_{3}, \widetilde{C}_{3} \in \mathbf{G}^{+}$;

(iv) $C=Y C_{4}, \widetilde{C}=Y \widetilde{C}_{4}$ with $C_{4}, \widetilde{C}_{4} \in \mathbf{G}^{+}$.

In the case of (i), we have

$$
X A=X C_{1} X^{2} D, X B=X \widetilde{C}_{1} \widetilde{D} \quad \text { or } \quad X A=X C_{1} Y^{2} D, X B=X \widetilde{C}_{1} \widetilde{D} .
$$


Multiplying these equalities by $t^{-3} Y X^{2} Y X$ from the left yields

$$
A=C_{1} X^{2} D, B=\widetilde{C}_{1} \widetilde{D} \quad \text { or } \quad A=C_{1} Y^{2} D, B=\widetilde{C}_{1} \widetilde{D}
$$

This implies that $A \Rightarrow_{1} B$. In the case of (ii), we see that $X C_{2}, Y \widetilde{C}_{2} \in \boldsymbol{\Lambda}$ since $X C_{2} \sim Y \widetilde{C}_{2}$, and hence there exists $\bar{C}_{2} \in \mathbf{G}^{+}$such that $\widetilde{C}=Y \widetilde{C}_{2}=X \bar{C}_{2}$. Therefore it is reduced to the case of (i). We can treat the case of (iii) similarly to the case of (ii). In the case of (iv), we see that $X A, X B \in \boldsymbol{\Lambda}$ and hence there exist $\widetilde{A}, \widetilde{B} \in \mathbf{G}^{+}$such that

$$
X A=X Y X \widetilde{A} \quad \text { and } \quad X B=X Y X \widetilde{B} .
$$

Then it follows that

$$
A=Y X \widetilde{A} \quad \text { and } \quad B=Y X \widetilde{B}
$$

We also get

$$
Y X Y \widetilde{A}=Y C_{4} X^{2} D, Y X Y \widetilde{B}=Y \widetilde{C}_{4} \widetilde{D} \text { or } Y X Y \widetilde{A}=Y C_{4} Y^{2} D, Y X Y \widetilde{B}=Y \widetilde{C}_{4} \widetilde{D}
$$

and hence

$$
X Y \widetilde{A}=C_{4} X^{2} D, X Y \widetilde{B}=\widetilde{C}_{4} \widetilde{D} \quad \text { or } \quad X Y \widetilde{A}=C_{4} Y^{2} D, X Y \widetilde{B}=\widetilde{C}_{4} \widetilde{D}
$$

This yields $X Y \widetilde{A} \Rightarrow_{1} X Y \widetilde{B}$. Since $\ell(Y \widetilde{B})=k$, we have $Y \widetilde{A} \Rightarrow_{1} Y \widetilde{B}$. Since $\ell(\widetilde{B})=k$, we have $\widetilde{A} \Rightarrow_{1} \widetilde{B}$ and hence $A \Rightarrow_{1} B$ from (3.7). The second statement of (3.6) is similarly shown.

We next suppose that

$$
\left\{\begin{array}{l}
X A \Rightarrow X B \quad \Rightarrow \quad A \Rightarrow B, \\
Y A \Rightarrow Y B \Rightarrow A \Rightarrow B
\end{array}\right.
$$

if $\ell(A)-\ell(B) \leq 2 m$. Let $X A \Rightarrow X B$ and $\ell(A)-\ell(B)=2(m+1)$. Then there exist $A_{1}, A_{2}, \ldots, A_{m} \in \mathbf{G}^{+}$such that

$$
X A \Rightarrow_{1} A_{m} \Rightarrow_{1} A_{m-1} \Rightarrow_{1} \cdots \Rightarrow_{1} A_{1} \Rightarrow_{1} X B \text {. }
$$

It is easily seen that if $A_{j}=X \widetilde{A}_{j}$ with some $\widetilde{A}_{j} \in \mathbf{G}^{+}$for some $1 \leq j \leq m$, then $A \Rightarrow A_{j}$ and $A_{j} \Rightarrow B$ and hence $A \Rightarrow B$ since $\ell(A)-\ell\left(A_{j}\right) \leq 2 m$ and $\ell\left(A_{j}\right)-\ell(B) \leq 2 m$. Therefore we may assume that $A_{j}=Y \bar{A}_{j}$ for some $\bar{A}_{j} \in \mathbf{G}^{+}$ and $Y \bar{A}_{j} \notin \boldsymbol{\Lambda}$ for $j=1,2, \ldots, m$.

We show that (3.8) with $\ell(A)-\ell(B)=2(m+1)$ holds if $\ell(B)=1$. Let $X A \Rightarrow X B$ for such $A, B$. If $B=Y$, then $Y \bar{A}_{1} \in \boldsymbol{\Lambda}$. This contradiction implies that $B=X$. Then it follows that

$$
Y \bar{A}_{n}=Y^{2 i_{1}} X^{2 j_{1}} Y^{2 i_{2}} X^{2 j_{2}} \cdots Y^{2 i_{N(n)}} X^{2 j_{N(n)}}
$$

for some nonnegative integers $i_{\nu}, j_{\nu}$ for $1 \leq \nu \leq N(n)$ with $2\left(i_{1}+j_{1}+i_{2}+j_{2}+\right.$ $\left.\cdots+i_{N(n)}+j_{N(n)}\right)=2(n+1)$ for $1 \leq n \leq m$. Indeed, it is trivial for $n=1$ since $Y \bar{A}_{1}=Y^{2} X^{2}$. Suppose that (3.9) is valid for $n$. From $Y \bar{A}_{n+1} \Rightarrow_{1} Y \bar{A}_{n}$, there exist $C^{*}, C_{*}, D^{*}, D_{*} \in \mathbf{G}^{+}$with $C^{*} \sim C_{*}$ and $D^{*} \sim D_{*}$ such that

$$
Y \bar{A}_{n+1}=C^{*} X^{2} D^{*}, Y \bar{A}_{n}=C_{*} D_{*} \quad \text { or } \quad Y \bar{A}_{n+1}=C^{*} Y^{2} D^{*}, Y \bar{A}_{n}=C_{*} D_{*} .
$$

Since $Y \bar{A}_{n}$ cannot be equivalently deformed to a different form, (3.9) for $n+1$ is only the possibility for $Y \bar{A}_{n+1}$ to satisfy $Y \bar{A}_{n+1} \notin \boldsymbol{\Lambda}$. Therefore (3.9) is true for $1 \leq n \leq m$.

There exists $\underline{A} \in \mathbf{G}^{+}$such that $X A=X Y X^{2} \underline{A}$. In fact, $X A$ is equivalent to a representation $Z$ obtained by setting $X^{2}$ or $Y^{2}$ somewhere in $Y \bar{A}_{m}$ so that $X A \in \boldsymbol{\Lambda}$ 
by the same argument as above. If $X^{2}$ or $Y^{2}$ is set in $Y^{2 i_{1}-2} X^{2 j_{1}} \cdots Y^{2 i_{N(m)}} X^{2 j_{N(m)}}$ $\in \boldsymbol{\Lambda}$ with $i_{1} \geq 2$ in $Z$ so that $Y^{2 i_{1}-2} X^{2 j_{1}} \cdots Y^{2 i_{N(m)}} X^{2 j_{N(m)}}=X Y X A^{\prime}$ for some $A^{\prime} \in \mathbf{G}^{+}$, then

$$
X A=Y^{2} X Y X A^{\prime}=Y X Y X^{2} A^{\prime}=X Y X^{3} A^{\prime} .
$$

If not so, then $j_{1} \geq 1$ and

$$
\begin{aligned}
X A & =Y X^{2} Y X^{2 j_{1}} Y^{2 i_{2}} X^{2 j_{2}} \cdots Y^{2 i_{N}(m)} X^{2 j_{N}(m)} \\
& =Y X Y X Y X^{2 j_{1}-1} Y^{2 i_{2}} X^{2 j_{2}} \cdots Y^{2 i_{N(m)}} X^{2 j_{N(m)}} \\
& =X Y X^{2} Y X^{2 j_{1}-1} Y^{2 i_{2}} X^{2 j_{2}} \cdots Y^{2 i_{N(m)}} X^{2 j_{N(m)}}
\end{aligned}
$$

Therefore it follows that $Y^{2} X Y \underline{A}=X Y X^{2} \underline{A}=X A \Rightarrow Y \bar{A}_{1}=Y^{2} X^{2}$. Since $\ell\left(Y^{2} X Y \underline{A}\right)-\ell\left(Y^{2} X^{2}\right)=2 m$, we get $Y \underline{A} \Rightarrow X$. Since $X A=X Y X^{2} \underline{A}$, we obtain $A=Y X^{2} \underline{A} \Rightarrow_{1} Y \underline{A} \Rightarrow X=B$.

Suppose that (3.8) with $\ell(A)-\ell(B)=2(m+1)$ is true if $\ell(B) \leq k$. Let $X A \Rightarrow X B$ and $\ell(B)=k+1$. Since $Y \bar{A}_{1} \Rightarrow_{1} X B$, there exist $E, \widetilde{E}, F, \widetilde{F} \in \mathbf{G}^{+}$ with $E \sim \widetilde{E}$ and $F \sim \widetilde{F}$ such that

$$
Y \bar{A}_{1}=E X^{2} F, X B=\widetilde{E} \widetilde{F} \quad \text { or } \quad Y \bar{A}_{1}=E Y^{2} F, X B=\widetilde{E} \widetilde{F} .
$$

If $E=X E_{1}$ with $E_{1} \in \mathbf{G}^{+}$, then $Y \bar{A}_{1}=X E_{1} X^{2} F$, which contradicts $Y \bar{A}_{1} \notin \mathbf{\Lambda}$. Therefore we get $E=Y E_{2}$ with $E_{2} \in \mathbf{G}^{+}$. Then $\widetilde{E}$ divides into two cases:

(I) $\widetilde{E}=X \widetilde{E}_{2}$ with $\widetilde{E}_{2} \in \mathbf{G}^{+}$;

(II) $\widetilde{E}=Y \widetilde{E}_{3}$ with $\widetilde{E}_{3} \in \mathbf{G}^{+}$.

In the case of $(\mathrm{I})$, we see that $Y E_{2} \in \boldsymbol{\Lambda}$ from $Y E_{2} \sim X \widetilde{E}_{2}$, and hence $Y E_{2}=X \bar{E}_{2}$ for some $\bar{E}_{2} \in \mathbf{G}^{+}$. Then it follows that $Y \bar{A}_{1}=X \bar{E}_{2} X^{2} F$ or $Y \bar{A}_{1}=X \bar{E}_{2} Y^{2} F$. This contradiction excludes the case of (I).

In the case of (II), we have $X B=Y X Y \bar{B}$ for some $\bar{B} \in \mathbf{G}^{+}$by $X B \in \mathbf{\Lambda}$. Since $X A \Rightarrow_{1} Y \bar{A}_{m}$, there exist $P, Q, \widetilde{P}, \widetilde{Q} \in \mathbf{G}^{+}$with $P \sim \widetilde{P}$ and $Q \sim \widetilde{Q}$ such that

$$
X A=P X^{2} Q, Y \bar{A}_{m}=\widetilde{P} \widetilde{Q} \quad \text { or } \quad X A=P Y^{2} Q, Y \bar{A}_{m}=\widetilde{P} \widetilde{Q} .
$$

If $\widetilde{P}=X P_{1}$ for some $P_{1} \in \mathbf{G}^{+}$, then $Y \bar{A}_{m} \in \mathbf{\Lambda}$. This contradiction implies that $\widetilde{P}=Y \widetilde{P}_{2}$ with $\widetilde{P}_{2} \in \mathbf{G}^{+}$. Then we have two cases:

(1) $P=X P_{3}$ with $P_{3} \in \mathbf{G}^{+}$;

(2) $P=Y P_{4}$ with $P_{4} \in \mathbf{G}^{+}$.

In the case of (1), we see that $\widetilde{P} \in \boldsymbol{\Lambda}$, which contradicts $Y \bar{A}_{m} \notin \boldsymbol{\Lambda}$. Thus only the case of (2) is possible. Then we have $X A=Y P_{4} X^{2} Q$ or $X A=Y P_{4} Y^{2} Q$ and hence $X A=Y X Y \bar{A}$ with some $\bar{A} \in \mathbf{G}^{+}$. Therefore, $Y X Y \bar{A} \Rightarrow_{1} Y \bar{A}_{m} \Rightarrow_{1}$ $\cdots \Rightarrow_{1} Y \bar{A}_{1} \Rightarrow_{1} Y X Y \bar{B}$ and hence $X Y \bar{A} \Rightarrow_{1} \bar{A}_{m} \Rightarrow_{1} \cdots \Rightarrow_{1} \bar{A}_{1} \Rightarrow_{1} X Y \bar{B}$. Since $\ell(Y \bar{B})=k$, we get $Y \bar{A} \Rightarrow Y \bar{B}$ and $\ell(Y \bar{A})-\ell(Y \bar{B})=2(m+1)$, which implies that $\bar{A} \Rightarrow \bar{B}$ since $\ell(\bar{B})=k-1$. Thus $A=Y X \bar{A} \Rightarrow Y X \bar{B}=B$.

We can similarly show the second assertion of (3.8). This completes the proof of the first statement of this lemma. The second statement is proved in the same say as above.

We prove the following result on specific braids.

Lemma 3.2. Let $A_{2 k}=\left(X Y^{2} X\right)^{k} Y^{2 k+1}$ and $B_{2 k}=X^{2} Y^{2 k+1} X^{2 k}$ for a positive integer $k$. Then $A_{2 k} \nRightarrow B_{2 k}$. 

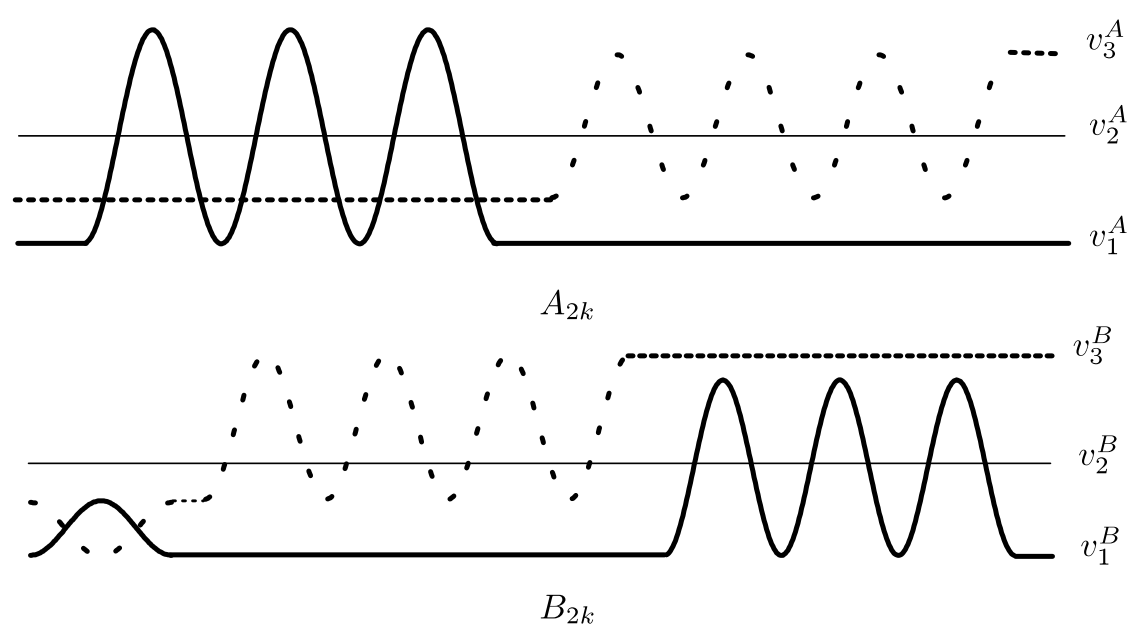

Figure 3.4 AND FiguRE 3.5

Proof. We first get

$$
A_{2 k}=\left(X Y^{2} X\right)^{k-1} X Y^{2} X Y^{2} Y^{2(k-1)+1}=t^{3} A_{2(k-1)}
$$

by (3.4).

It follows from (3.4) that

$$
\begin{aligned}
A_{2} \Rightarrow B_{2} & \Leftrightarrow A_{2}=B_{2} \\
& \Leftrightarrow X Y^{2} X Y^{3}=X^{2} Y^{3} X^{2} \\
& \Leftrightarrow t^{3} Y=X^{2} Y^{3} X^{2} \\
& \Leftrightarrow t^{3} Y^{2} X^{2}=X^{2} Y^{2} Y X^{2} Y X^{2} \\
& \Leftrightarrow t^{3} Y^{2} X^{2}=t^{3} X^{2} Y^{2} .
\end{aligned}
$$

Since $Y^{2} X^{2} \neq X^{2} Y^{2}$ for $t \neq \pm 1$ by straightforward calculation, we see that $A_{2} \not \nexists$ $B_{2}$.

On the contrary, we assume that $A_{2 n} \Rightarrow B_{2 n}$ for some $n \geq 2$. Let

$$
k=\min \left\{1 \leq i \leq n: A_{2 i} \Rightarrow B_{2 i}\right\} .
$$

$A_{2 k}$ and $B_{2 k}$ are represented as three strands $v_{1}^{A}, v_{2}^{A}, v_{3}^{A}$ and $v_{1}^{B}, v_{2}^{B}, v_{3}^{B}$, respectively, in Figures 3.4 and 3.5. Here the figures are in the case of $k=3$ for simplicity.

Then there exists a mapping $\left(v_{1}, v_{2}, v_{3}\right):\left[S_{1}, S_{2}\right] \rightarrow \mathbf{G}^{+}$with $S_{1}<S_{2}$ such that

(i) $v_{i}\left(S_{1}\right)=v_{i}^{A}$ and $v_{i}\left(S_{2}\right)=v_{i}^{B}$ for $i=1,2,3$.

(ii) Denoting by $\mathcal{S}$ the set of $s \in\left[S_{1}, S_{2}\right]$ such that any zero of $v_{i}(s)-v_{j}(s)$ is nondegenerate for $i, j=1,2,3$ with $i \neq j,\left[S_{1}, S_{2}\right] \backslash \mathcal{S}$ is at most a finite set. Let $G(s)$ be a positive braid of $v_{1}(s), v_{2}(s), v_{3}(s)$ for $s \in \mathcal{S}$. For each $\underline{s}, \bar{s} \in \mathcal{S}$ with $\underline{s}<\bar{s}$, we have $G(\underline{s}) \sim G(\bar{s})$ or $G(\underline{s}) \Rightarrow G(\bar{s})$.

Denote by $\sigma_{i}(s)$ the $i$ th intersection between $v_{1}(s)$ and $v_{2}(s)$ for $i=1,2, \ldots, 2 k$ and by $\widetilde{\sigma}_{j}(s)$ the $j$ th intersection between $v_{2}(s)$ and $v_{3}(s)$ for $j=1,2, \ldots, 2 k+1$ for $s \in \mathcal{S}$. Here intersections are enumerated from the left. Let $s_{1} \in\left(S_{1}, S_{2}\right)$ such that $\tilde{\sigma}_{2 k}\left(s_{1}\right)=\sigma_{1}\left(s_{1}\right)$ and $\widetilde{\sigma}_{2 k}(s)<\sigma_{1}(s)$ for $s \in\left(s_{1}, S_{2}\right]$, and let $s_{2} \in\left(S_{1}, S_{2}\right)$ such that 


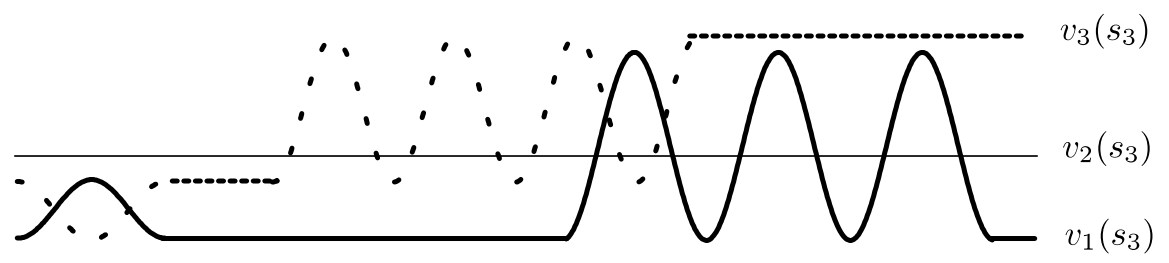

FiguRE 3.6

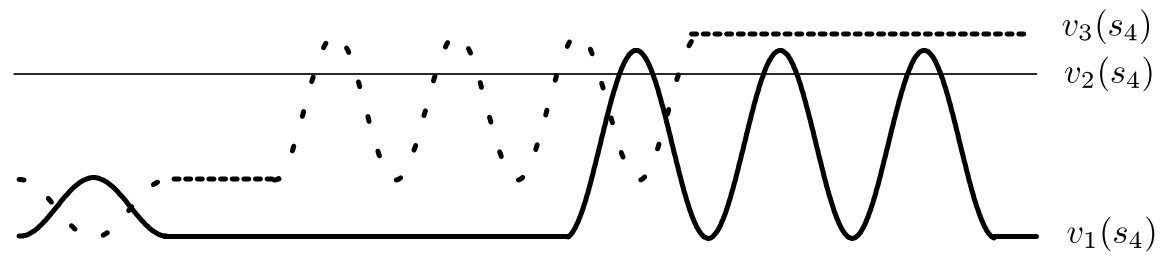

Figure 3.7

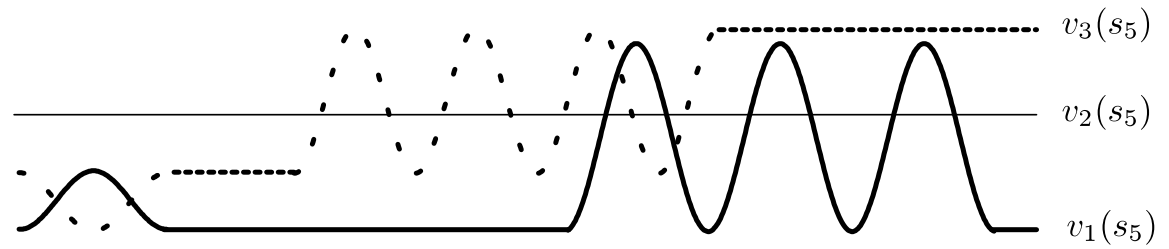

FiguRE 3.8

$\tilde{\sigma}_{2 k+1}\left(s_{2}\right)=\sigma_{2}\left(s_{2}\right)$ and $\tilde{\sigma}_{2 k+1}(s)<\sigma_{2}(s)$ for $s \in\left(s_{2}, S_{2}\right]$. In the case of $s_{1}>s_{2}$, there exists $s_{3} \in \mathcal{S}$ with $s_{2}<s_{3}<s_{1}$ such that (see Figure 3.6)

$$
A_{2 k} \Rightarrow G\left(s_{3}\right) \Rightarrow X^{2} Y^{2 k-1} X Y X^{2} Y X X^{2 k-2} \text {. }
$$

Since $X Y X^{2} Y X=(X Y)^{3}=t^{3} I$, we get $A_{2 k} \Rightarrow t^{3} B_{2(k-1)}$.

In the case of $s_{1}<s_{2}$, there exists $s_{4} \in \mathcal{S}$ with $s_{1}<s_{4}<s_{2}$ such that (see Figure 3.7)

$$
A_{2 k} \Rightarrow G\left(s_{4}\right) \Rightarrow X^{2} Y^{2 k-1} Y X Y^{2} X Y X^{2 k-2}=t^{3} B_{2(k-1)} .
$$

In the case of $s_{1}=s_{2}$, there exists $s_{5} \in \mathcal{S}$ with $S_{1}<s_{5}<s_{1}$ such that (see Figure 3.8)

$$
A_{2 k} \Rightarrow G\left(s_{5}\right) \Rightarrow X^{2} Y^{2 k-1} X Y X Y X Y X^{2 k-2}=t^{3} B_{2(k-1)} .
$$

In all the cases, we have $A_{2 k} \Rightarrow t^{3} B_{2(k-1)}$. Then it follows from (3.10) that $A_{2(k-1)} \Rightarrow B_{2(k-1)}$ by Lemma 3.1. This contradicts the definition of $k$, which completes the proof. 
Lemma 3.3. For a positive integer $k$, let $A_{2 k}, B_{2 k}$ be as in Lemma 3.2 and let $\widetilde{A}_{2 k}=\left(X Y^{2} X\right)^{k} Y^{2 k}, \widetilde{A}_{2 k+1}=\left(X Y^{2} X\right)^{k} X Y X^{2 k+1}, \widetilde{B}_{2 k}=X^{2} Y^{2 k} X Y^{2 k} X$ and $\widetilde{B}_{2 k+1}=X^{2} Y^{2 k+1} X^{2 k+1} Y$. Then $\widetilde{A}_{2 k} \nRightarrow \widetilde{B}_{2 k}$ and $\widetilde{A}_{2 k+1} \nRightarrow \widetilde{B}_{2 k+1}$.

Proof. If $\widetilde{A}_{2 k} \Rightarrow \widetilde{B}_{2 k}$, then we have $\left(X Y^{2} X\right)^{k} Y^{2 k} \Rightarrow X^{2} Y^{2 k} X Y^{2 k} X$. Then it follows from (3.3) that

$$
\left(X Y^{2} X\right)^{k} Y^{2 k+1} \Rightarrow X^{2} Y^{2 k} X Y^{2 k} X Y=X^{2} Y^{2 k} X^{2} Y X^{2 k} \Rightarrow X^{2} Y^{2 k+1} X^{2 k},
$$

which implies $A_{2 k} \Rightarrow B_{2 k}$. Since $A_{2 k} \nRightarrow B_{2 k}$ by Lemma 3.2 , we get $\widetilde{A}_{2 k} \nRightarrow \widetilde{B}_{2 k}$.

If $\widetilde{A}_{2 k+1} \Rightarrow \widetilde{B}_{2 k+1}$, then we get

$$
\left(X Y^{2} X\right)^{k} X Y X^{2 k+1} \Rightarrow X^{2} Y^{2 k+1} X^{2 k+1} Y .
$$

It follows from (3.1) and (3.3) that

$$
\left(X Y^{2} X\right)^{k} X Y X^{2 k+1}=\left(X Y^{2} X\right)^{k} Y^{2 k} X Y X=\left(X Y^{2} X\right)^{k} Y^{2 k+1} X Y=A_{2 k} X Y
$$

and

$$
X^{2} Y^{2 k+1} X^{2 k+1} Y=X^{2} Y^{2 k+1} X^{2 k} X Y=B_{2 k} X Y .
$$

Therefore, $A_{2 k} \Rightarrow B_{2 k}$ from Lemma 3.1. Since $A_{2 k} \not B_{2 k}$ by Lemma 3.2. we get $\widetilde{A}_{2 k+1} \nRightarrow \widetilde{B}_{2 k+1}$.

Lemma 3.3 can be shown directly by an argument similar to the proof of Lemma 3.2. but it is easier to make use of Lemma 3.2. Lemmas 3.1] 3.3 are shown by different methods in [10].

\section{Proof of the MAIN TheOREMS}

The present section is devoted to the proofs of Theorems 1.1 and 1.2 ,

Proof of Theorem 1.1. Let $w$ be the solution of (1.8) or (1.9) corresponding to $u$. We may assume without loss of generality that any zero of $w(s)-\varphi_{\infty}$ does not disappear for all $s \geq s^{T}$. On the contrary, suppose that

$$
\limsup _{s \rightarrow \infty} e^{-\gamma_{m} s}|w(s)|_{\infty}=\infty
$$

For $\mathcal{T}>0$, denote by $w_{\ell, \mathcal{T}}$ a solution obtained in Proposition 2.3 with $T$ replaced by $\mathcal{T}$.

Put $w_{m}=w_{m, T}$, and denote by $\widetilde{w}_{m}$ the solution of (1.8) defined by (2.13) with $W=w_{m, \widetilde{T}}$ for $0<\widetilde{T}<T$. From Propositions 2.1, 2.3, we see that

$$
\limsup _{s \rightarrow \infty} \frac{w(0, s)}{w_{m}(0, s)}=\infty .
$$

By Lemma 2.3, there exist $s_{1} \geq s^{T}, C>0$ and $d>0$ such that for $\tau \geq 2 \log d+C$,

(i) $w(s)-\varphi_{\infty}$ has $m$ zeros in $\left[0, d e^{s / 2}\right)$ for $s \geq s_{1}$;

(ii) $w\left(d e^{s / 2}, s\right)<\widetilde{w}_{m}\left(d e^{s / 2}, s+\tau\right)$ for $s \in\left[s_{1}, \widetilde{S}-\tau\right)$ if $m$ is even, and $w\left(d e^{s / 2}, s\right)$ $>\widetilde{w}_{m}\left(d e^{s / 2}, s+\tau\right)$ for $s \in\left[s_{1}, \widetilde{S}-\tau\right)$ if $m$ is odd, where $\widetilde{S}=-\log (T-\widetilde{T})$. 


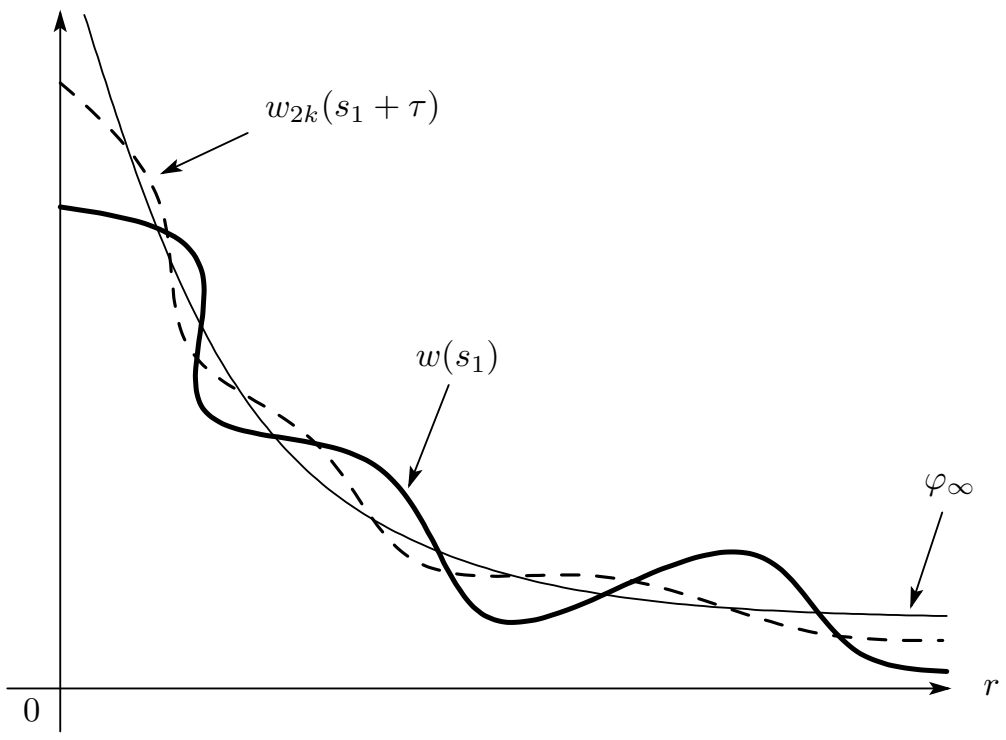

FiguRE 4.1

Suppose that $m$ is even, i.e., $m=2 k$ with positive integer $k$. Let $r_{i}(s)$ be the $i$ th zero of $w(s)-\varphi_{\infty}$ for a positive integer $i$. Moreover for sufficiently small $\varepsilon>0$ there exists a sufficiently large $\tau>0$ such that (see Figure 4.1)

$$
\left\{\begin{array}{c}
(I-i) w\left(0, s_{1}\right)<w_{2 k}\left(0, s_{1}+\tau\right) \\
(I-i i) \sup \left\{\left|w_{2 k}\left(r, s_{1}+\tau\right)-\varphi_{\infty}(r)\right|: r \in\left[r_{1}\left(s_{1}\right) / 2, d e^{s_{1} / 2}\right]\right\} \\
<\varepsilon \sup \left\{\left|w\left(r, s_{1}\right)-\varphi_{\infty}(r)\right|: r \in\left[r_{1}\left(s_{1}\right) / 2, d e^{s_{1} / 2}\right]\right\} ; \\
(I-i i i) w\left(s_{1}\right)-w_{2 k} \text { has } 2 k \text { zeros in }\left[0, d e^{s_{1} / 2}\right] .
\end{array}\right.
$$

It follows from (4.1) that there exists $s_{2}>s_{1}$ such that (see Figure 4.2)

$$
w\left(0, s_{2}\right)>w_{2 k}\left(0, s_{2}+\tau\right) .
$$

If $0<\widetilde{T}<T$ is sufficiently close to $T$, then (4.2) and (4.3) hold with $w_{2 k}$ replaced by $\widetilde{w}_{2 k}$. Since $\widetilde{w}_{2 k}(s+\tau)$ blows up at $s=\widetilde{S}-\tau$, there exists $s_{3}>s_{2}$ such that (see Figure 4.3)

(II-i): $\quad w\left(0, s_{3}\right)<\widetilde{w}_{2 k}\left(0, s_{3}+\tau\right)$;

(II-ii): $2 k$ zeros of $\widetilde{w}_{2 k}\left(s_{3}+\tau\right)-\varphi_{\infty}$ are located in $\left[0, r_{1}\left(s_{3}\right)\right)$.

Let us translate the situation to the framework of the braid group. For $s \in$ $\left[S_{1}, S_{2}\right]$ with $S_{1}=s_{1}$ and $S_{2}=s_{3}$, put $a(s)=0, b(s)=d e^{s / 2}, v_{1}(s)=w(s)$, $v_{2}(s)=\widetilde{w}_{2 k}(s+\tau)$ and $v_{3}(s)=\varphi_{\infty}$. We can draw these three solutions at $s=S_{1}$ as in Figure 4.4.

It follows from (3.1) that the braid of Figure 4.4 is equivalent to $\widetilde{A}_{2 k}$ in Lemma 3.3,

The braid of Figure 4.6 which is represented by $\widetilde{B}_{2 k}$ in Lemma 3.3 becomes a parabolic reduction of the braid corresponding to $v_{1}\left(S_{2}\right), v_{2}\left(S_{2}\right), v_{3}\left(S_{2}\right)$. In fact, at least two zeros of $w(s)-\widetilde{w}_{2 k}(s+\tau)$ which disappear at $r=0$ give no effects to other parts. Moreover other zeros of $w(s)-\widetilde{w}_{2 k}(s+\tau)$ may disappear at $r=0$, but their 


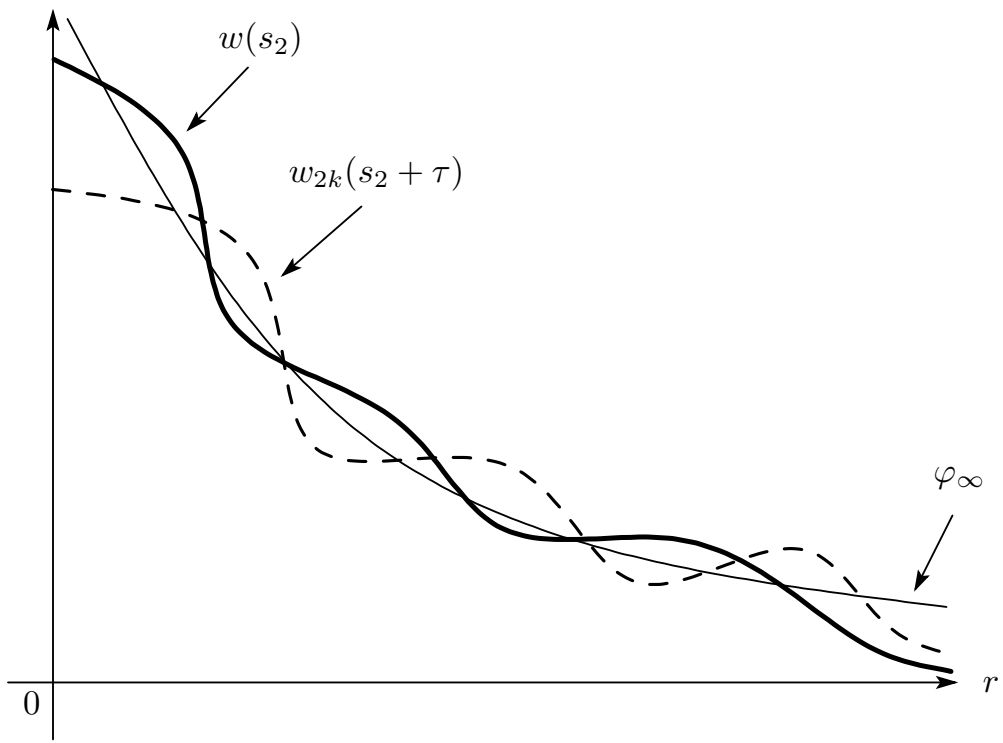

Figure 4.2

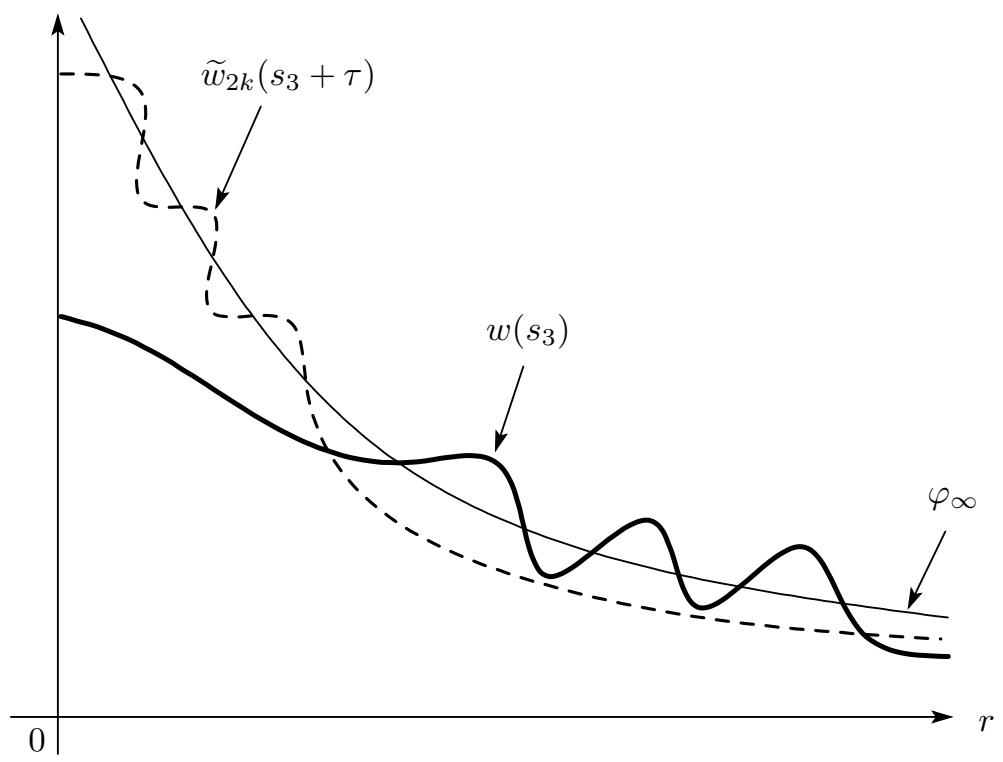

FiguRE 4.3

number is even. We regard them as a parabolic reduction. By this consideration, we obtain that $\widetilde{A}_{2 k} \Rightarrow \widetilde{B}_{2 k}$.

When $m$ is odd, i.e., $m=2 k+1$ with positive integer $k$, we can proceed similarly to the above. The braid at $s=S_{1}$ is equivalent to $\widetilde{A}_{2 k+1}$, and $\widetilde{B}_{2 k+1}$ is a parabolic 


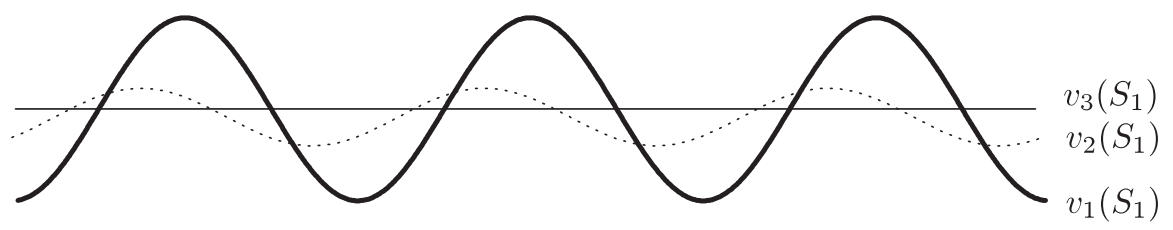

FiguRe 4.4

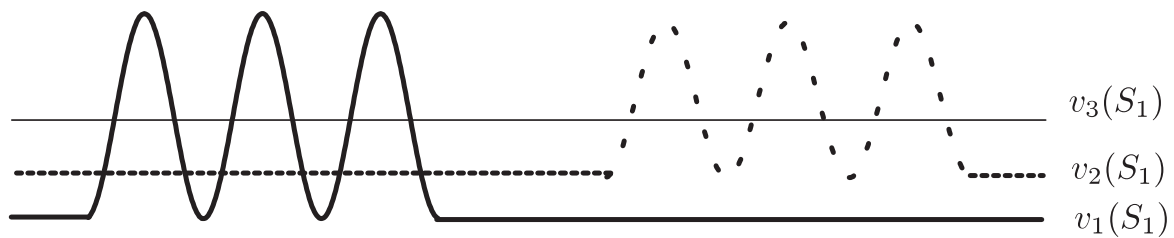

FIGURE 4.5

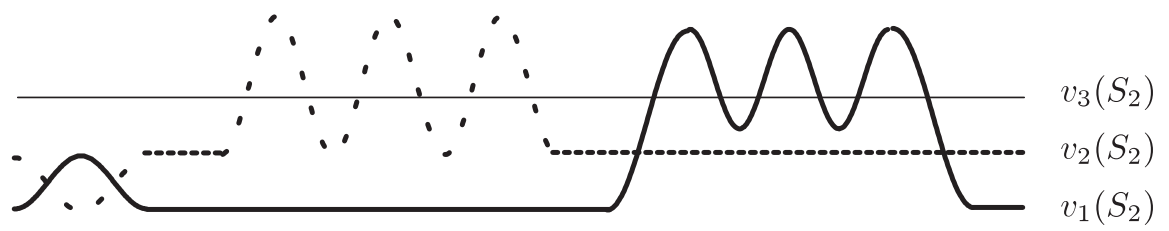

Figure 4.6

reduction of the braid at $s=S_{2}$, where $\widetilde{A}_{2 k+1}$ and $\widetilde{B}_{2 k+1}$ are as in Lemma 3.3. Then it follows that $\widetilde{A}_{2 k+1} \Rightarrow \widetilde{B}_{2 k+1}$

On the other hand, we see that $\widetilde{A}_{2 k} \nRightarrow \widetilde{B}_{2 k}$ and $\widetilde{A}_{2 k+1} \nRightarrow \widetilde{B}_{2 k+1}$ by Lemma 3.3 . This contradiction completes the proof.

In the proof of Theorem 1.1, the device based on three solutions $w, \widetilde{w}_{m}(\cdot+\tau), \varphi$ was introduced in [17. We regard $r=-\infty$ as a virtual boundary point in the proof of Theorem 1.1. We get the same conclusion if we extend solutions to $(-\infty,+\infty)$ by reflection at $r=0$ and consider symmetric braids with respect to $r=0$ defined on $[-b(s), b(s)]$.

Proof of Theorem 1.2. In all the cases, we may assume without loss of generality that any zero of $w(s)-\varphi_{\infty}$ does not disappear for $s \geq s^{T}$. We first treat the Cauchy problem. Let $w$ be the solution of (1.8) corresponding to $u$ for which the conclusion is false. For $0<\widetilde{T}<T$, put $\widetilde{u}(\xi, t)=\mu^{1 /(p-1)} u\left(\mu^{1 / 2} \xi, \mu t\right)$ for $t \in[0, \widetilde{T})$ with $\mu=T / \widetilde{T}$. Denote by $\widetilde{w}$ the solution of (1.8) corresponding to $\widetilde{u}$ blowing up at $s=\widetilde{S} \equiv-\log (\widetilde{T}-T)$. Suppose that $m=2 k$ for a positive integer $k$. Let $w_{2 k}$ be a solution of (1.8) obtained in Proposition 2.3 with $\ell=2 k$ such that $w_{2 k}\left(s^{T}\right)$ has compact support. Then there exists $\tau>0$ such that if $0<\widetilde{T}<T$ is sufficiently close to $T$, then $z\left(\widetilde{w}\left(s^{T}+\tau\right)-w_{2 k}\left(s^{T}\right)\right)=2 k$. Replacing $w$ and $\widetilde{w}_{2 k}(\cdot+\tau)$ by $w_{2 k}$ and $\widetilde{w}(\cdot+\tau)$, respectively, and putting $a(s) \equiv 0$ and $b(s) \equiv \infty$ in the proof of Theorem 1.1, we obtain (1.18) with some constant $\underline{C}>0$. 
We next suppose that $m=2 k+1$ for a positive integer $k$. If $m \geq q+1$, then $\lambda_{m-1}>0$ by Proposition 2.2 and hence there exist $\tau>0$ and a solution $w_{2 k}$ of (1.8) obtained in Proposition 2.3 with $\ell=2 k$ such that $z\left(\widetilde{w}\left(s^{T}+\tau\right)-w_{2 k}\left(s^{T}\right)\right)=2 k$ if $0<\widetilde{T}<T$ is sufficiently close to $T$. Replacing $w$ and $\widetilde{w}_{2 k}(\cdot+\tau)$ by $w_{2 k}$ and $\widetilde{w}(\cdot+\tau)$, respectively, and putting $a(s) \equiv 0$ and $b(s) \equiv 0$ in the proof of Theorem 1.1. the braid at $s=S_{1}$ is equivalent to $A_{2 k}$, and $B_{2 k}$ is parabolic reduction of the braid at $s=S_{2}$, where $A_{2 k}$ and $B_{2 k}$ are as in Lemma 3.2. This implies that $A_{2 k} \Rightarrow B_{2 k}$, which contradicts Lemma 3.2. Thus (1.19) is valid for some $C_{1}>0$.

Under the second hypothesis, we may suppose without loss of generality that $t_{0}=0$. Take a solution $w_{2 k+1}$ of (1.8) obtained in Proposition 2.3 with initial data $w_{2 k+1}\left(s^{T}\right)$ satisfying

$$
w_{2 k+1}\left(r, s^{T}\right) \geq(1+2 \varepsilon) c_{\infty} r^{-\frac{2}{p-1}} \quad \text { for } r \geq e^{\widetilde{\sigma} s^{T}}+1,
$$

where $\widetilde{\sigma}$ is the constant in Remark 2.3. Then there exist $\tau>0$ and $0<\widetilde{T}<T$ such that $\widetilde{w}(s+\tau)-\varphi_{\infty}$ and $\widetilde{w}\left(s^{T}+\tau\right)-w_{2 k+1}\left(s^{T}\right)$ have $2 k+1$ zeros in $\left[0, b e^{s+\tau}\right)$ and

$$
\widetilde{w}\left(b e^{s+\tau}, s+\tau\right)<w_{2 k+1}\left(b e^{s+\tau}, s\right) \quad \text { for } s \in\left[s^{T}, \widetilde{S}-\tau\right) .
$$

Replacing $w$ and $w_{2 k}(\cdot+\tau)$ by $w_{2 k+1}$ and $\widetilde{w}(\cdot+\tau)$ and putting $a(s)=0$ and $b(s)=b e^{s+\tau}$ for $s \in\left[s^{T}, \widetilde{S}-\tau\right)$ in the proof of Theorem 1.1, we obtain (1.18) with some constant $\underline{C}>0$.

In the case of the Cauchy-Dirichlet problem, let $w$ be the solution of (1.9) corresponding to $u$. When $m=2 k$ for a positive integer $k$, there exists $\tau>0$ such that if $0<\widetilde{T}<T$ is sufficiently close to $T$, then $\widetilde{w}\left(s^{T}+\tau\right)-w_{2 k}\left(s^{T}\right)$ has $m$ zeros in $\left[0, \delta\left(s^{T}+\tau\right)\right)$ and another zero close to $c e^{\left(s^{T}+\tau\right) / 2}$, and

$$
\widetilde{w}(\delta(s+\tau), s+\tau)>w_{2 k}(\delta(s+\tau), s) \quad \text { for } s \in\left[s^{T}, \widetilde{S}-\tau\right),
$$

where $0<\delta(s)<c e^{s / 2}$ for $s \in\left[s^{T}, \widetilde{S}-\tau\right)$. Replacing $w$ and $\widetilde{w}_{2 k}(\cdot+\tau)$ by $w_{2 k}$ and $\widetilde{w}(\cdot+\tau)$, respectively, and putting $a(s)=0$ and $b(s)=\delta(s+\tau)$ for $s \in\left[S_{1}, S_{2}\right]$ in the proof of Theorem 1.1, we get (1.18) with some constant $\underline{C}>0$. The proof can be shown similarly in the case of odd $m$. This completes the proof.

\section{REMARK}

In this section, we show that Lemma 3.3 is optimal in the following sense.

Proposition 5.1. Let $C_{2 k-1}=\left(X Y^{2} X\right)^{k} Y^{2 k-1}, C_{2 k}=\left(X Y^{2} X\right)^{k} X Y X^{2 k}, D_{2 k-1}$ $=X^{2} Y^{2 k-1} X^{2 k}$ and $D_{2 k}=X^{2} Y^{2 k} X Y^{2 k+1}$ for a positive integer $k$. Then $C_{2 k-1} \Rightarrow$ $D_{2 k}$ and $C_{2 k} \Rightarrow D_{2 k}$.

Proof. By (3.2) and (3.3), the following hold:

(i) $\left(X Y^{2} X\right)^{k+1} Y^{2}=\left(X Y^{2} X\right)^{k} Y^{2} X Y^{2} X$ for any nonnegative integer $k$;

(ii) $X Y X Y X Y^{2 k}=Y^{2 k} X Y X Y X$ and $Y X Y X Y X^{2 k}=X^{2 k} Y X Y X Y$ for any positive integer $k$. 
From (3.1), it is immediate that $C_{1} \Rightarrow D_{1}$. If $C_{2 k-1} \Rightarrow D_{2 k-1}$, then we have

$$
\begin{aligned}
\left(X Y^{2} X\right)^{k+1} Y^{2(k+1)-1} & =\left(X Y^{2} X\right)^{k+1} Y^{2} Y^{2 k-1} \\
& =\left(X Y^{2} X\right)^{k} Y Y^{2(k-1)} X Y X Y X Y \\
& \Rightarrow X^{2} Y^{2 k-1} X^{2 k} X Y X Y X Y \\
& =X^{2} Y^{2 k-1} X Y X Y X Y X^{2 k} \\
& \Rightarrow X^{2} Y^{2(k+1)-1} X^{2(k+1)}
\end{aligned}
$$

by (3.2), (3.3), (i) and (ii). This implies that $C_{2(k+1)-1} \Rightarrow D_{2(k+1)-1}$. Therefore it follows that $C_{2 k-1} \Rightarrow D_{2 k-1}$ for each positive integer $k$.

Since $C_{2 k-1} \Rightarrow D_{2 k-1}$, it follows from (3.3) that

$$
C_{2 k}=\left(X Y^{2} X\right)^{k} Y^{2 k-1} Y X Y \Rightarrow X^{2} Y^{2 k-1} X^{2 k} Y X Y=D_{2 k} .
$$

This completes the proof.

We applied braid group theory to prove the analytic results in Theorems 1.1 and 1.2. On the other hand, an algebraic result, Proposition 5.1, can be easily shown by the analytic method.

Analytic proof of Proposition 5.1. Let $p>p_{L}$ so that $\lambda_{j}>0$ for $j \geq 2$. For $\mathcal{T}$, denote by $w_{\ell, \mathcal{T}}$ a solution obtained in Proposition 2.3 with $T$ replaced by $\mathcal{T}$. Take $m \geq 3$. For sufficiently small $\varepsilon>0$ there exist $w_{m} \equiv w_{m, T}, w_{m-1} \equiv w_{m-1, T}$, $s_{1} \geq s^{T}$ and $\tau>0$ such that

$$
\left\{\begin{array}{l}
(I-i) w_{m}\left(0, s_{1}\right)<w_{m-1}\left(0, s_{1}+\tau\right) ; \\
(I-i i)\left|w_{m-1}\left(r, s_{1}+\tau\right)-\varphi_{\infty}(r)\right|<\varepsilon\left|w_{m}\left(r, s_{1}\right)-\varphi_{\infty}(r)\right|, r \in\left[\frac{R_{1}\left(s_{1}\right)}{2}, d e^{s_{1} / 2}\right] ; \\
(I-i i i) z\left(w_{m}\left(s_{1}\right)-w_{m-1}\left(s_{1}+\tau\right)\right)=m,
\end{array}\right.
$$

where $R_{i}(s)$ is the $i$ th zero of $w_{m}(s)-\varphi_{\infty}$ for a positive integer $i$. From Proposition 2.3. there exists $s_{2}>s_{1}$ such that

$$
w_{m}\left(0, s_{2}\right)>w_{m-1}\left(0, s_{2}+\tau\right) .
$$

Let $\widetilde{w}_{m-1}$ be the solution defined in (2.13) with $W=w_{m-1, \widetilde{T}}$ for $0<\widetilde{T}<T$. If $0<\widetilde{T}<T$ is sufficiently close to $T$, then (5.1) and (5.2) hold with $w_{m-1}$ replaced by $\widetilde{w}_{m-1}$. Since $\widetilde{w}_{m-1}(s+\tau)$ blows up at $s=\widetilde{S}-\tau$ with $\widetilde{S}=-\log (T-\widetilde{T})$, there exists $s_{3} \in\left(s_{2}, \widetilde{S}-\tau\right)$ such that

(II-i) $\quad w\left(0, s_{3}\right)<\widetilde{w}_{m-1}\left(0, s_{3}+\tau\right)$;

(II-ii) $m-1$ zeros of $\widetilde{w}_{m-1}\left(s_{3}+\tau\right)-\varphi_{\infty}$ are located in $\left[0, R_{1}\left(s_{3}\right)\right)$.

Put $S_{1}=s_{1}, S_{2}=s_{3}, a(s) \equiv 0$ and $b(s) \equiv+\infty$. In the case of $m=2 k$ with a positive integer $k$, let $v_{1}=w_{2 k}, v_{2}=\varphi_{\infty}$ and $v_{3}=\widetilde{w}_{2 k-1}(\cdot+\tau)$ and argue similarly to the proof of Theorem 1.1. The braid of $v_{1}\left(S_{1}\right), v_{2}\left(S_{1}\right), v_{3}\left(S_{1}\right)$ is equivalent to $C_{2 k-1}$, and $D_{2 k-1}$ is a parabolic reduction of the braid of $v_{1}\left(S_{2}\right), v_{2}\left(S_{2}\right), v_{3}\left(S_{2}\right)$. This implies that $C_{2 k-1} \Rightarrow D_{2 k-1}$. When $m=2 k+1$ with a positive integer $k$, let $v_{1}=\widetilde{w}_{2 k}(\cdot+\tau), v_{2}=\varphi_{\infty}$ and $v_{3}=w_{2 k+1}$. We similarly obtain $C_{2 k} \Rightarrow D_{2 k}$. This completes the proof. 


\section{REFERENCES}

[1] S. Fillippas and R. V. Kohn, Refined asymptotics for the blow-up of $u_{t}-\Delta u=u^{p}$, Comm. Pure Appl. Math. 45 (1992), 821-869. MR1164066 (93g:35066)

[2] R. W. Ghrist, J. B. Van den Berg and R. C. Vandervorst, Morse theory on spaces of braids and Lagrangian dynamics, Invent. Math. 152 (2003), 369-432. MR1974892 (2004e:37025)

[3] R. W. Ghrist and R. C. Vandervorst, Braids and scalar parabolic PDEs. In: Proceedings of New Directions in Dynamics Systems, Kyoto, 2002.

[4] Y. Giga and R. V. Kohn, Characterizing blowup using similarity variables, Indiana Univ. Math. J. 36 (1987), 1-40. MR876989 (88c:35021)

[5] M. A. Herrero and J. J. L. Velázquez, Blow-up behaviour of one-dimensional semilinear parabolic equations, Ann. Inst. H. Poincaré 10 (1993), 131-189. MR1220032 (94g:35030)

[6] Explosion de solutions des équations paraboliques semilinéaires supercritiques, C. R. Acad. Sci. Paris 319 (1994), 141-145.

[7] _ A blow up result for semilinear heat equations in the supercritical case, preprint.

[8] Y. Li, Asymptotic behavior of positive solutions of equation $\Delta u+K(x) u^{p}=0$ in $\mathbf{R}^{N}, \mathrm{~J}$. Differential Equations 95 (1992), 304-330. MR1165425 (93k:35048)

[9] H. Matano, Blow-up in nonlinear heat equations with supercritical power nonlinearity, Contemp. Math. 446 (2007), 385-412. MR2376669 (2008m:35186)

[10] _ Parabolic reduction of braids and its application to nonlinear heat equations, in preparation.

[11] H. Matano and F. Merle, On nonexistence of type II blowup for a supercritical nonlinear heat equation, Comm. Pure Appl. Math. 57 (2004), 1494-1541. MR2077706 (2005e:35115)

[12] - Classification of Type I and Type II behaviors for a supercritical nonlinear heat equation, J. Functional Anal. 256 (2009), 992-1064. MR2488333 (2009k:35124)

[13] T. Matsuoka, Braid invariants and instability of periodic solutions of time-periodic 2dimensional ODE's, Topol. Methods Nonlinear Anal. 14 (1999), 261-274. MR1766186 (2001e:37032)

[14] N. Mizoguchi, Blow-Up behavior of solutions for a semilinear heat equation with supercritical nonlinearity, J. Differential Equations 205 (2004), 298-328. MR2092860 (2005j:35106)

[15] _ Type-II blow-up for a semilinear heat equation, Adv. Differential Equations 9 (2004), 1279-1316. MR2099557(2005k:35204)

[16] B B Bundedness of global solutions for a supercritical semilinear heat equation and its application, Indiana Univ. Math. J. 54 (2005), 1047-1059. MR2164417 (2006e:35174)

[17] _ Rate of type II blow-up for a semilinear heat equation, Math. Ann. 339 (2007), 839-877. MR2341904 (2008k:35246)

[18] J. J. L. Velázquez, Classification of singularities for blowing up solutions in higher dimensions, Trans. Amer. Math. Soc. 338 (1993), 441-464. MR1134760 (93j:35101)

Department of Mathematics, Tokyo Gakugei University, Koganei, Tokyo 184-8501, Japan - and - Precursory Research for Embryonic Science and Technology, Japan Science and Technology Agency, 4-1-8 Honcho Kawaguchi, Saitama 332-0012, Japan

E-mail address: mizoguti@u-gakugei.ac.jp 